\title{
DIMENSION CONSERVATION FOR SELF-SIMILAR SETS AND FRACTAL PERCOLATION
}

\author{
KENNETH FALCONER AND XIONG JIN
}

\begin{abstract}
We introduce a technique that uses projection properties of fractal percolation to establish dimension conservation results for sections of deterministic self-similar sets. For example, let $K$ be a self-similar subset of $\mathbb{R}^{2}$ with Hausdorff dimension $\operatorname{dim}_{H} K>1$ such that the rotational components of the underlying similarities generate the full rotation group. Then for all $\epsilon>0$, writing $\pi_{\theta}$ for projection onto the line $L_{\theta}$ in direction $\theta$, the Hausdorff dimensions of the sections satisfy $\operatorname{dim}_{H}\left(K \cap \pi_{\theta}^{-1} x\right)>\operatorname{dim}_{H} K-1-\epsilon$ for a set of $x \in L_{\theta}$ of positive Lebesgue measure, for all directions $\theta$ except for those in a set of Hausdorff dimension 0. For a class of self-similar sets we obtain a similar conclusion for all directions, but with lower box dimension replacing Hausdorff dimensions of sections. We obtain similar inequalities for the dimensions of sections of Mandelbrot percolation sets.
\end{abstract}

\section{INTRODUCTION}

Relating the Hausdorff dimension $\operatorname{dim}_{H} K$ of a set $K \subset \mathbb{R}^{d}$ to the dimensions of its sections and projections has a long history. The best-known result on projections is that, if $K$ is Borel or analytic, then, writing $\pi_{V}: \mathbb{R}^{d} \rightarrow V$ for orthogonal projection onto the subspace $V$,

$$
\operatorname{dim}_{H} \pi_{V} K=\min \left(k, \operatorname{dim}_{H} K\right),
$$

for almost all $k$-dimensional subspaces $V$ (with respect to the natural invariant measure on subspaces). For sections of sets, for almost all $k$-dimensional subspaces $V$, the dimensions of the sections or slices $\pi_{V}^{-1} x \cap K$ of $K$ satisfy

$$
\operatorname{dim}_{H}\left(K \cap \pi_{V}^{-1} x\right) \leq \max \left(0, \operatorname{dim}_{H} K-k\right)
$$

for Lebesgue almost all $x \in V$ (we take $\operatorname{dim}_{H} \emptyset=-\infty$ ). Moreover, for all $\epsilon>0$ and almost all $V$, there is a set $W_{\epsilon} \subset V$ of positive $k$-dimensional Lebesgue measure such that

$$
\operatorname{dim}_{H}\left(K \cap \pi_{V}^{-1} x\right) \geq \max \left(0, \operatorname{dim}_{H} K-k\right)-\epsilon
$$

for $x \in W_{\epsilon}$. These inequalities were obtained by Marstrand [15] for subsets of the plane, and extended to general $d$ and $k$ by Mattila [17. Kaufman [12] introduced the potential theoretic method which is now commonly used in studying dimensions of projections and sections of sets.

These properties are complemented by the fact [16] that, for all $k$-dimensional subspaces $V$, for all $0 \leq \Delta \leq d-k$,

$$
\Delta+\operatorname{dim}_{H}\left\{x \in V: \operatorname{dim}_{H}\left(K \cap \pi_{V}^{-1} x\right) \geq \Delta\right\} \leq \operatorname{dim}_{H} K .
$$

In particular, if $\operatorname{dim}_{H} K>k$ then for all $V$

$$
\operatorname{dim}_{H}\left(K \cap \pi_{V}^{-1} x\right) \leq \operatorname{dim}_{H} K-k
$$


for Lebesgue almost all $x \in V$. A good exposition of this material may be found in [18.

Fursternberg [8] introduced the notion of dimension conservation: given $K \subset \mathbb{R}^{d}$, a projection $\pi_{V}$ is said to be dimension conserving for $K$ if there is a number $\Delta>0$ such that

$$
\Delta+\operatorname{dim}_{H}\left\{x \in V: \operatorname{dim}_{H}\left(K \cap \pi_{V}^{-1} x\right) \geq \Delta\right\} \geq \operatorname{dim}_{H} K
$$

In this paper we consider a slightly weaker property when $\operatorname{dim}_{H} K>k$. We say that a projection $\pi_{V}$ is weakly dimension conserving if, for all $\epsilon>0$,

$$
\operatorname{dim}_{H}\left(K \cap \pi_{V}^{-1} x\right)>\operatorname{dim}_{H} K-k-\epsilon \quad \text { for all } x \in W
$$

where $W$ is a 'large' subset of $V$, either with $\operatorname{dim}_{H} W=k$ or with $\mathcal{L}^{k}(W)>0$, where $\mathcal{L}^{k}$ denotes $k$-dimensional Lebesgue measure. It follows from $\sqrt{1.2}$ that $\pi_{V}$ is weakly dimension conserving for almost every $k$-dimensional subspace $V$.

There has been great interest recently in identifying classes of sets, in particular classes of self-similar sets and their variants, for which these various inequalities hold for all, rather than just almost all, subspaces. Several papers establish (1.1) for all projections for classes of self-similar sets [8, 10, 21, 25] and for percolation on self-similar sets $[7,22,23,24,26$. Here we consider dimensions of sections, and identify sets for which (1.4), or a similar inequality for box-counting dimension, holds for all subspaces $V$.

Recall that an iterated function system (IFS) $\mathcal{I}=\left\{f_{i}\right\}_{i=1}^{m}$ on $\mathbb{R}^{d}$ is a family of $2 \leq m<\infty$ contractions $f_{i}: \mathbb{R}^{d} \rightarrow \mathbb{R}^{d}$. An IFS determines a unique non-empty compact $K \subset \mathbb{R}^{d}$ such that

$$
K=\bigcup_{i=1}^{m} f_{i}(K),
$$

called the attractor of the IFS, see [6, 11]. If the $f_{i}$ are all similarities then $K$ is self-similar. The IFS satisfies the strong separation condition (SSC) if the union (1.5) is disjoint, and the open set condition (OSC) if there is a non-empty open set $U$ such that $\cup_{i=1}^{m} f_{i}(U) \subset U$ with this union disjoint. If either SSC or OSC hold then $\operatorname{dim}_{H} K=s$ where $s$ is given by $\sum_{i=1}^{m} r_{i}^{s}=1$, where $r_{i}$ is the similarity ratio of $f_{i}$.

We may write an IFS of (orientation preserving) similarities as

$$
\mathcal{I}=\left\{f_{i}=r_{i} R_{i} \cdot+a_{i}\right\}_{i=1}^{m}
$$

where $R_{i} \in S O(d, \mathbb{R})$ is a rotation, $r_{i}$ is the scaling ratio and $a_{i}$ is a translation. If the group $G$ generated by $\left\{R_{1}, \ldots, R_{m}\right\}$ is dense in $S O(d, \mathbb{R})$ we say that the IFS has dense rotations.

A number of results on dimension conservation of self-similar sets have been established. Furstenberg 8 showed that (1.3) holds for projections onto all subspaces $V$ for a class of 'homogeneous' sets. These include self-similar sets where the IFS $\mathcal{I}$ consists of contracting homotheties (i.e. similarities without rotation or reflection so that $R_{i}=I$ for all $i$ ) that satisfy SSC or OSC. For example, variants on the Sierpiński carpet are of this type, where the value of $\Delta$ in (1.3) depends on the subspace $V$. There are detailed analyses of sections of the Sierpiński carpet in 14, 13] and of sections of the Sierpiński gasket or triangle in [2]. In the case where the IFS $\mathcal{I}$ satisfies OSC and the group generated by $\left\{R_{1}, \ldots, R_{m}\right\}$ is finite, 
then every projection is dimension conserving, that is for all $V$ (1.3) holds for some number $\Delta$, see [7, 9].

In this paper we demonstrate that many self-similar sets $K$ are weakly dimension conserving for all, or virtually all, projections $\pi_{V}$. For self-similar sets in $\mathbb{R}^{2}$ where $\mathcal{I}$ satisfies OSC and has dense rotations and $\operatorname{dim}_{H} K>1,(1.4)$ holds with $\mathcal{L}(W)>0$ for all $\epsilon>0$ and for projections onto all lines $V$, except for lines in a set of directions of Hausdorff dimension 0 (Theorem 4.8). Provided that we replace Hausdorff dimension by lower box dimension on the left-hand side of the inequality we get (1.4) for all lines, for a large class of sets that satisfy a projection condition (Theorem 3.2). We also show that, almost surely, (1.4) is true for all $k$-dimensional subspaces $V$ for random subsets of $\mathbb{R}^{d}$ obtained by the Mandelbrot percolation process (Theorem 3.4).

The idea is to demonstrate weak dimension conservation for a deterministic set $K$ by running a percolation-type process on $K$ to 'probe' the dimensions of its sections. We construct random sets $K^{\omega} \subset K$ such that $k<\operatorname{dim} K^{\omega}<k+\epsilon / 2$ with positive probability. Writing $L_{x}$ for the $(d-k)$-plane through $x$ and perpendicular to $V$, if $\operatorname{dim}\left(K \cap L_{x}\right)<\operatorname{dim} K-k-\epsilon$ for some $x \in V$ there is a high probability that $K^{\omega} \cap L_{x}=\emptyset$ or equivalently that $x \notin \pi_{V} K^{\omega}$. By invoking results on projections of random sets that show that with positive probability $\operatorname{dim} \pi_{V} K^{\omega}=k$, we conclude that there must be a significant subset of $x \in V$, indeed a subset of dimension $k$, for which this does not occur.

We formulate this principle in a general context in Propositions 2.1 and 2.2 . To apply it in various settings we utilise results on dimensions of projections of percolation sets from [7, 22, 23, 24]. Theorems 4.6 and 4.8 depend on the absolute continuity of projections of an alternative type of random measure, and this is established in Theorem 4.5 which is a random version of a deterministic result of Shmerkin and Solomyak [25].

The authors are grateful to Mike Hochman for comments on an earlier version of this paper.

\section{ESTIMATES FOR DIMENSIONS OF SECTIONS USING RANDOM SUBSETS}

In this section we present a general formulation of our method for obtaining lower bounds for the dimensions of sections of a set given a knowledge of the dimensions of projections of related random subsets. The method applies to sets that can be modeled in terms of an infinite rooted tree. These include self-similar sets, where the tree provides a natural description of the hierarchical construction of the set, but extends to a many further fractals.

Let $\Lambda=\{1, \ldots, m\}$ be an alphabet of $m \geq 2$ symbols, with $\Lambda^{n}$ denoting the set of words of length $n \geq 0$. Let $\Sigma_{*}:=\cup_{n \geq 0} \Lambda^{n}$ be the set of finite words and $\Sigma:=\Lambda^{\mathbb{N}}$ the corresponding symbolic space of all infinite words. For each $\mathbf{i} \in \Sigma_{*}$ denote by $[\mathbf{i}] \subset \Sigma$ the set of infinite words that start with $\mathbf{i}$, that is the cylinder rooted at $\mathbf{i}$. We denote the diameter of a set $A \subset \mathbb{R}^{d}$ by $|A|$.

We consider fractals which are the image of a subset of symbolic space under a continuous mapping $\Phi: \Sigma \mapsto \mathbb{R}^{d}$ with the usual metrics. For each $\mathbf{i} \in \Sigma_{*}$ we write $B(\Phi[\mathbf{i}])$ for the closed convex hull of $\Phi[\mathbf{i}]$. We also assume throughout that there is a number $d_{0}>0$ such that

$$
\frac{\text { inradius } B(\Phi[\mathbf{i}])}{\text { diameter } B(\Phi[\mathbf{i}])} \geq d_{0} \quad \text { for all } \mathbf{i} \in \Sigma_{*}
$$


thus the convex hulls cannot get 'too long and thin'. We assume throughout that $\Phi$ satisfies the following two conditions:

(1) There exist $0<c_{0}, c_{1}<\infty$ such that for all $\rho \in\left(0, c_{0}\right)$, the set

$$
\Lambda_{\rho}=\left\{\mathbf{i} \in \Sigma_{*}: \rho \leq|\Phi[\mathbf{i}]|<c_{1} \rho\right\}
$$

yields a finite covering of $\Sigma$, that is $\# \Lambda_{\rho}<\infty$ and $\Sigma=\cup_{\mathbf{i} \in \Lambda_{\rho}}[\mathbf{i}]$;

(2) There exists an integer $n_{0}$ such that for all $\rho \in\left(0, c_{0}\right)$ and $x \in \mathbb{R}^{n}$,

$$
\#\left\{\mathbf{i} \in \Lambda_{\rho}: x \in B(\Phi[\mathbf{i}])\right\} \leq n_{0} .
$$

These conditions will certainly be satisfied if $\Phi$ codes the attractor of an IFS satisfying OSC.

We may define measures of Hausdorff type on subsets of $\Phi(\Sigma)$ by setting, for all $s>0, F \subset \Phi(\Sigma)$ and $\delta>0$,

$$
\mathcal{M}_{\delta}^{s}(F)=\inf \left\{\sum_{j=1}^{\infty}\left|\Phi\left[\mathbf{i}_{j}\right]\right|^{s}: \Phi^{-1}(F) \subset \bigcup_{j=1}^{\infty}\left[\mathbf{i}_{j}\right],\left|\Phi\left[\mathbf{i}_{j}\right]\right| \leq \delta\right\}
$$

and

$$
\mathcal{M}^{s}(F)=\lim _{\delta \searrow 0} \mathcal{M}_{\delta}^{s}(F) .
$$

Then $\mathcal{M}^{s}$ is equivalent to the restriction of $s$-dimensional Hausdorff measure $\mathcal{M}^{s}$ to $\Phi(\Sigma)$. Clearly $\mathcal{H}^{s}(F) \leq \mathcal{M}^{s}(F)$ for $F \subset \Phi(\Sigma)$. For the opposite inequality (to within a constant multiple), note that the number of sets $\Phi[\mathbf{i}]$ with $\mathbf{i} \in \Lambda_{\rho}$ that overlap $U \cap \Phi(\Sigma)$ is bounded for all $U \subset \mathbb{R}^{n}$ with $|U|=\rho<c_{0}$, from comparing the volumes of maximal inscribed balls of $B(\Phi[\mathbf{i}])$ with that of some ball centered in $U$ of radius $|U|$ and using $(2.2)$. In particular, $\operatorname{dim}_{H} F=\inf \left\{s: \mathcal{M}^{s}(F)=0\right\}=$ $\sup \left\{s: \mathcal{M}^{s}(F)=\infty\right\}$ for $F \subset \Phi(\Sigma)$.

In a similar way, 2.1) and (2.2) imply that the box-counting dimension of subsets of $\Phi(\Sigma)$ may be found by counting cylinders. In particular, the lower box-counting dimension of $F \subset \Phi(\Sigma)$ is given by

$$
\underline{\operatorname{dim}}_{B} F=\varliminf_{\rho \rightarrow 0} \frac{\log \left\{\# \mathbf{i} \in \Lambda_{\rho}: F \cap B(\Phi[\mathbf{i}]) \neq 0\right\}}{-\log \rho} .
$$

Let $\mathcal{B}_{\Sigma}$ be the $\sigma$-field generated by the cylinders of $\Sigma$. Let $\mathbb{P}$ be a probability measure on $\mathcal{B}_{\Sigma}$. Let $\Sigma^{\omega}$ be a random subset of $\Sigma$ and let

$$
\Sigma_{*}^{\omega}:=\left\{\mathbf{i} \in \Sigma_{*}:[\mathbf{i}] \cap \Sigma^{\omega} \neq \emptyset\right\} .
$$

We adopt the convention that $A^{\omega}:=A \cap \Sigma^{\omega}$ if $A$ is a subset of $\Sigma$ and $A^{\omega}:=A \cap \Sigma_{*}^{\omega}$ if $A$ is a subset of $\Sigma_{*}$.

For $\alpha \geq 0$ we say that $\Sigma^{\omega}$ is an $\alpha$-random subset of $\Sigma$ if there exists a constant $c_{2}<\infty$ such that for all $\rho \in\left(0, c_{0}\right)$ and all $\mathbf{i} \in \Lambda_{\rho}$,

$$
\mathbb{P}\left(\mathbf{i} \in \Lambda_{\rho}^{\omega}\right) \leq c_{2} \rho^{\alpha} .
$$

For our applications, $\Sigma^{\omega}$ will typically be the symbolic set underlying fractal percolation on $K$, so that $\Phi\left(\Sigma^{\omega}\right)=K^{\omega}$.

Let $V$ be a $k$-dimensional subspace of $\mathbb{R}^{d}$ and let $\pi_{V}: \mathbb{R}^{d} \rightarrow V$ denote orthogonal projection onto $V$. Write $\mathcal{L}^{k}$ for $k$-dimensional Lebesgue measure on $V$ identified with $\mathbb{R}^{k}$ in the obvious way. (If $k=1$ then $V$ is a line and we write $\mathcal{L}$ for Lebesgue measure on $V$.) 
The following two propositions are our principal tools. The first, which concerns the Hausdorff measure of sections, has stronger hypotheses on the projection of the random subset but a weaker condition on the projection of the original set, than the second which concerns the lower box dimension of sections.

Proposition 2.1. Let $A \in \mathcal{B}_{\Sigma}$. Let $\Sigma^{\omega}$ be an $\alpha$-random subset of $\Sigma$ for some $\alpha>0$, let $\Phi: \Sigma \rightarrow \mathbb{R}^{d}$ satisfy (1) and (2) above, and let $V$ be a $k$-dimensional subspace of $\mathbb{R}^{d}$. If $\mathbb{P}\left(\mathcal{L}^{k}\left(\pi_{V}\left(\Phi\left(A^{\omega}\right)\right)\right)>0\right)>0$, then

$$
\mathcal{L}^{k}\left\{x \in V: \operatorname{dim}_{H}\left(\Phi(A) \cap \pi_{V}^{-1}(x)\right) \geq \alpha\right\}>0 .
$$

Proof. Let

$$
S=\left\{x \in V: \operatorname{dim}_{H}\left(\Phi(A) \cap \pi_{V}^{-1}(x)\right)<\alpha\right\} .
$$

Let $x \in S$. Using $(2.3)$, for all $\epsilon>0$ we may find a set of words $\mathcal{J} \subset \Sigma_{*}$ such that $\Phi^{-1}\left(\Phi(A) \cap \pi_{V}^{-1}(x)\right) \subset \bigcup_{\mathbf{i} \in \mathcal{J}}[\mathbf{i}]$ and $\sum_{\mathbf{i} \in \mathcal{J}}|\Phi[\mathbf{i}]|^{\alpha}<\epsilon$. Then $\Phi\left(A^{\omega}\right) \cap \pi_{V}^{-1}(x) \subset$ $\bigcup_{\mathbf{i} \in \mathcal{J} \cap \Sigma_{*}^{\omega}} \Phi[\mathbf{i}]$ and

$$
\mathbb{E}\left(\#\left\{\mathbf{i} \in \mathcal{J} \cap \Sigma_{*}^{\omega}\right\}\right)=\sum_{\mathbf{i} \in \mathcal{J}} \mathbb{P}\left(\mathbf{i} \in \Sigma_{*}^{\omega}\right) \leq c_{2} \sum_{\mathbf{i} \in \mathcal{J}}|\Phi[\mathbf{i}]|^{\alpha}<c_{2} \epsilon,
$$

using 2.5 , so $\mathbb{P}\left(\left\{\mathbf{i} \in \mathcal{J} \cap \Sigma_{*}^{\omega}\right\} \neq \emptyset\right)<c_{2} \epsilon$. Since $\epsilon$ is arbitrarily small, we conclude that for all $x \in S, \Phi\left(A^{\omega}\right) \cap \pi_{V}^{-1}(x)=\emptyset$ almost surely.

By Fubini's theorem, almost surely

$$
\mathcal{L}^{k}\left(S \cap \pi_{V}\left(\Phi\left(A^{\omega}\right)\right)\right)=\mathcal{L}^{k}\left(x \in S: \Phi\left(A^{\omega}\right) \cap \pi_{V}^{-1}(x) \neq 0\right)=0 .
$$

Hence, with positive probability,

$$
0<\mathcal{L}^{k}\left(\pi_{V}\left(\Phi\left(A^{\omega}\right)\right)\right)=\mathcal{L}^{k}\left(\pi_{V}\left(\Phi\left(A^{\omega}\right)\right) \backslash S\right) \leq \mathcal{L}^{k}\left(\pi_{V}(\Phi(A)) \backslash S\right) .
$$

The second general proposition concerns the lower box-counting dimension of sections of sets. Here we require a condition that, for all $\mathbf{i} \in \Sigma_{*}$, the projection of $\Phi[\mathbf{i}]$ onto the subspace $V$ is the same as that of its convex hull; in particular this will be the case if $\Phi[\mathbf{i}]$ is connected.

Proposition 2.2. Let $\Sigma^{\omega}$ be an $\alpha$-random subset of $\Sigma$ for some $\alpha>0$, let $\Phi: \Sigma \rightarrow$ $\mathbb{R}^{d}$ satisfy (1) and (2) above, and let $V$ be a line, that is a 1-dimensional subspace of $\mathbb{R}^{d}$. Suppose that the projection of $\Phi[\mathbf{i}]$ onto $V$ is the same as that of its convex hull $B(\Phi[\mathbf{i}])$ for all $\mathbf{i} \in \Sigma_{*}$. If $\mathbb{P}\left(\operatorname{dim}_{H} \pi_{V}\left(\Phi\left(\Sigma^{\omega}\right)\right)=1\right)>0$, then for every $\epsilon \in(0, \alpha)$,

$$
\operatorname{dim}_{H}\left\{x \in V: \underline{\operatorname{dim}}_{B}\left(\Phi(\Sigma) \cap \pi_{V}^{-1}(x)\right)>\alpha-\epsilon\right\}=1 .
$$

Proof. To keep the notation simple, we give the proof for $\Phi: \Sigma \rightarrow \mathbb{R}^{2}$ where the sections are intersections with lines perpendicular to the line $V$. The proof is virtually identical for $\Phi: \Sigma \rightarrow \mathbb{R}^{d}$ where $d>2$. Write $L_{x} \equiv \pi_{V}^{-1}(x)$ for the line through $x \in V$ perpendicular to $V$. For $x \in V$ and $\rho \in\left(0, c_{0}\right)$ write

$$
N(x, \rho):=\#\left\{\mathbf{i} \in \Lambda_{\rho}: B(\Phi[\mathbf{i}]) \cap L_{x} \neq \emptyset\right\} \equiv \#\left\{\mathbf{i} \in \Lambda_{\rho}: \Phi[\mathbf{i}] \cap L_{x} \neq \emptyset\right\}
$$

for the 'box counting numbers', where the equivalence follows as every line that intersects the convex hull $B(\Phi[\mathbf{i}])$ also intersects $\Phi[\mathbf{i}]$.

Here is the first of three subsidiary lemmas within this proof. This enables us to reduce consideration of coverings of subsets of $L_{x}$ when estimating $N(x, \rho)$ to a small set of $x$. We identify $V$ with $\mathbb{R} \times\{0\} \subset \mathbb{R}^{2}$ in the obvious way. 
Lemma 2.3. Let $\rho \in\left(0, c_{0}\right)$ and $M>0$. Let $I \subset V$ be an interval with $|I| \leq \rho$ such that $N(x, \rho) \leq M$ for some $x \in I$. Then there exist $x_{1}, x_{2} \in I$ with $x_{1} \leq x_{2}$ such that

$$
N\left(x_{1}, \rho\right), N\left(x_{2}, \rho\right) \leq M
$$

and such that, if $x \in I$ has $N(x, \rho) \leq M$, then, for all $\mathbf{i} \in \Lambda_{\rho}$ such that $B(\Phi[\mathbf{i}]) \cap$ $L_{x} \neq \emptyset$, either $B(\Phi[\mathbf{i}]) \cap L_{x_{1}} \neq \emptyset$ or $B(\Phi[\mathbf{i}]) \cap L_{x_{2}} \neq \emptyset$.

Proof. Let $x_{1}^{\prime}=\inf \{x \in I: N(x, \rho) \leq M\}$. If $N\left(x_{1}^{\prime}, \rho\right) \leq M$ then take $x_{1}=x_{1}^{\prime}$. Otherwise take $x_{1}>x_{1}^{\prime}$ sufficiently close to $x_{1}^{\prime}$ to ensure that both $N\left(x_{1}, \rho\right) \leq M$ and

$$
\begin{aligned}
\left\{\mathbf{i} \in \Lambda_{\rho}:\right. & \left.B(\Phi[\mathbf{i}]) \cap L_{x_{1}} \neq \emptyset\right\} \\
& =\left\{\mathbf{i} \in \Lambda_{\rho}: B(\Phi[\mathbf{i}]) \cap L_{x_{1}} \neq \emptyset \text { and } \pi_{V}(\operatorname{int} B(\Phi[\mathbf{i}])) \cap\left[x_{1}, \infty\right) \neq \emptyset\right\} .
\end{aligned}
$$

In the same way, we may take $x_{2}$ to be $\sup \{x \in I: N(x, \rho) \leq M\}$ or a slightly smaller number if necessary. Clearly we may ensure that $x_{1} \leq x_{2}$. Since the $B(\Phi[\mathbf{i}])$ with $\mathbf{i} \in \Lambda_{\rho}$ have diameter at least $\rho$ and $x_{2}-x_{1} \leq \rho$, the conclusion of the lemma follows.

We now write

$$
N^{\omega}(x, \rho)=\#\left\{\mathbf{i} \in \Lambda_{\rho}^{\omega}: B(\Phi[\mathbf{i}]) \cap L_{x} \neq \emptyset\right\}
$$

for the random analogue of 2.6). Fix $\epsilon \in(0, \alpha)$ and for $\rho \in\left(0, c_{0}\right)$ let $S_{\rho}$ be the deterministic subset of $V$ :

$$
S_{\rho}=\left\{x \in V: N(x, \rho) \leq \rho^{-\alpha+\epsilon / 2}\right\} .
$$

The second subsidiary lemma shows that if $x \in S_{\rho}$ then the probability that $L_{x}$ has non-empty intersection with $\Phi\left(\Sigma^{\omega}\right)$ is small.

Lemma 2.4. Let $\rho \in\left(0, c_{0}\right)$ and let $I \subset V$ be an interval with $|I| \leq \rho$ such that $I \cap S_{\rho} \neq \emptyset$. Then

$$
\mathbb{P}\left(N^{\omega}(x, \rho)>0 \text { for some } x \in I \cap S_{\rho}\right) \leq 2 c_{2} \rho^{\epsilon / 2} .
$$

Proof. If $I \cap S_{\rho}=\emptyset$ then 2.8 is trivial. Otherwise, applying Lemma 2.3 to the interval $I$, taking $M=\rho^{-\alpha+\epsilon / 2}$ and noting (2.7), we may find $x_{1}, x_{2} \in I \cap S_{\rho}$ such that, for all $x \in I \cap S_{\rho}$, all $\omega$, and all $\mathbf{i} \in \Lambda_{\rho}^{\omega} \subset \Lambda_{\rho}$ with $B(\Phi[\mathbf{i}]) \cap L_{x} \neq \emptyset$, either $B(\Phi[\mathbf{i}]) \cap L_{x_{1}} \neq \emptyset$ or $B(\Phi[\mathbf{i}]) \cap L_{x_{2}} \neq \emptyset$. In particular, for all $x \in I \cap S_{\rho}$

$$
N^{\omega}(x, \rho) \leq N^{\omega}\left(x_{1}, \rho\right)+N^{\omega}\left(x_{2}, \rho\right) .
$$

For $j=1,2$, using 2.5 and 2.7,

$$
\begin{aligned}
\mathbb{E}\left(N^{\omega}\left(x_{j}, \rho\right)\right) & =\sum\left\{\mathbb{P}\left(\mathbf{i} \in \Lambda_{\rho}^{\omega}\right): \mathbf{i} \in \Lambda_{\rho}, B(\Phi[\mathbf{i}]) \cap L_{x_{j}} \neq \emptyset\right\} \\
& \leq \sum\left\{c_{2} \rho^{\alpha}: \mathbf{i} \in \Lambda_{\rho}, B(\Phi[\mathbf{i}]) \cap L_{x_{j}} \neq \emptyset\right\} \\
& \leq c_{2} \rho^{\alpha} N\left(x_{j}, \rho\right) \\
& \leq c_{2} \rho^{\alpha} \rho^{-\alpha+\epsilon / 2}
\end{aligned}
$$

so

$$
\mathbb{P}\left(N^{\omega}\left(x_{j}, \rho\right)>0\right) \leq c_{2} \rho^{\epsilon / 2} .
$$

The conclusion $(2.8)$ follows from 2.9 . 
Let

$$
S=\left\{x \in V: \underline{\operatorname{dim}}_{B}\left(\Phi(\Sigma) \cap L_{x}\right) \leq \alpha-\epsilon\right\} .
$$

Note that, for all $\rho \in\left(0, c_{0}\right)$, we have

$$
\Phi(\Sigma) \cap L_{x} \subset \bigcup_{\mathbf{i} \in \Lambda_{\rho}} \Phi[\mathbf{i}] \cap L_{x} .
$$

Thus, from (2.7), 2.6) and 2.4,

$$
S \subset \bigcap_{N=N_{0}}^{\infty} \bigcup_{n=N}^{\infty} S_{2^{-n}}
$$

where we choose $N_{0}$ so that $0<2^{-N_{0}}<c_{0}$.

The final subsidiary lemma essentially shows that the Hausdorff dimension of $S$ cannot be too big.

Lemma 2.5. With $S$ as above, $\operatorname{dim}_{H}\left(\pi_{V}\left(\Phi\left(\Sigma^{\omega}\right)\right) \cap S\right) \leq 1-\epsilon / 4$ almost surely.

Proof. For $\rho \in\left(0, c_{0}\right)$ write

$$
K_{\rho}^{\omega}:=\bigcup\left\{B(\Phi[\mathbf{i}]): \mathbf{i} \in \Lambda_{\rho}^{\omega}\right\} \supset \Phi\left(\Sigma^{\omega}\right) .
$$

Let $I \subset V$ be an interval with $|I|=\rho \leq c_{0}$. If $S_{\rho} \cap I \neq \emptyset$ then by Lemma 2.4

$$
\mathbb{P}\left(\pi_{V}\left(K_{\rho}^{\omega}\right) \cap S_{\rho} \cap I \neq \emptyset\right) \leq 2 c_{2} \rho^{\epsilon / 2} .
$$

For $n \geq N_{0}$, let $\mathcal{C}_{n}$ be the family of closed binary subintervals of $V$ of lengths $2^{-n}$. Thus, for $n \geq N_{0}$,

$$
\mathbb{E}\left(\# j: \pi_{V}\left(K_{2^{-n}}^{\omega}\right) \cap S_{2^{-n}} \cap I_{j} \neq \emptyset, I_{j} \in \mathcal{C}_{n}\right) \leq 2^{n+1}|\Phi(\Sigma)| 2 c_{2} 2^{-n \epsilon / 2}=c_{3} 2^{n(1-\epsilon / 2)} .
$$

In particular,

$$
\sum_{n=N_{0}}^{\infty} 2^{-n(1-\epsilon / 4)} \mathbb{E}\left(\# j: \pi_{V}\left(K_{2^{-n}}^{\omega}\right) \cap S_{2^{-n}} \cap I_{j} \neq \emptyset, I_{j} \in \mathcal{C}_{n}\right)=c_{3} \sum_{n=N_{0}}^{\infty} 2^{-\epsilon / 4}<\infty .
$$

Then, for all $N \geq N_{0}$,

$$
\begin{aligned}
\pi_{V}\left(\Phi\left(\Sigma^{\omega}\right)\right) \cap S & \subset \pi_{V}\left(\Phi\left(\Sigma^{\omega}\right)\right) \cap \bigcup_{n=N}^{\infty} S_{2^{-n}} \\
& =\bigcup_{n=N}^{\infty} \pi_{V}\left(\Phi\left(\Sigma^{\omega}\right)\right) \cap S_{2^{-n}} \\
& \subset \bigcup_{n=N}^{\infty} \pi_{V}\left(K_{2^{-n}}^{\omega}\right) \cap S_{2^{-n}} \\
& \subset \bigcup_{n=N}^{\infty} \bigcup_{I_{j} \in \mathcal{C}_{n}}\left\{I_{j}: \pi_{V}\left(K_{2^{-n}}^{\omega}\right) \cap S_{2^{-n}} \cap I_{j} \neq \emptyset\right\} .
\end{aligned}
$$

Hence, writing $\mathcal{H}_{\delta}^{s}$ for the $s$-dimensional Hausdorff $\delta$-premeasure, and $\mathcal{H}^{s}$ for $s$ dimensional Hausdorff measure, it follows on taking these covers of $\pi_{V}\left(\Phi\left(\Sigma^{\omega}\right)\right) \cap S$ 
for each $N$ that

$$
\begin{aligned}
\mathbb{E}\left(\mathcal { H } ^ { 1 - \epsilon / 4 } \left(\pi_{V}\right.\right. & \left.\left.\left(\Phi\left(\Sigma^{\omega}\right)\right) \cap S\right)\right) \\
& =\mathbb{E}\left(\lim _{N \rightarrow \infty} \mathcal{H}_{2^{-N}}^{1-\epsilon / 4}\left(\pi_{V}\left(\Phi\left(\Sigma^{\omega}\right)\right) \cap S\right)\right) \\
& \leq \limsup _{N \rightarrow \infty} \mathbb{E}\left(\mathcal{H}_{2^{-N}}^{1-\epsilon / 4}\left(\pi_{V}\left(\Phi\left(\Sigma^{\omega}\right)\right) \cap S\right)\right) \\
& \leq \mathbb{E}\left(\sum_{n=N_{0}}^{\infty} 2^{-n(1-\epsilon / 4)}\left(\# j: \pi_{V}\left(K_{2^{-n}}^{\omega}\right) \cap S_{2^{-n}} \cap I_{j} \neq \emptyset, I_{j} \in \mathcal{C}_{n}\right)\right) \\
& <\infty .
\end{aligned}
$$

It follows that almost surely $\mathcal{H}^{1-\epsilon / 4}\left(\pi_{V}\left(\Phi\left(\Sigma^{\omega}\right)\right) \cap S\right)<\infty$ and so $\operatorname{dim}_{H}\left(\pi_{V}\left(\Phi\left(\Sigma^{\omega}\right)\right) \cap\right.$ $S) \leq 1-\epsilon / 4$.

To complete the proof of Proposition 2.2, note that

$$
\operatorname{dim}_{H} \pi_{V}\left(\Phi\left(\Sigma^{\omega}\right)\right)=\max \left\{\operatorname{dim}_{H}\left(\pi_{V}\left(\Phi\left(\Sigma^{\omega}\right)\right) \cap S\right), \operatorname{dim}_{H}\left(\pi_{V}\left(\Phi\left(\Sigma^{\omega}\right)\right) \backslash S\right)\right\}
$$

so that, conditional on $\operatorname{dim}_{H} \pi_{V}\left(\Phi\left(\Sigma^{\omega}\right)\right)=1$, an event of positive probability by the hypothesis of the proposition,

$$
1 \leq \max \left\{1-\epsilon / 4, \operatorname{dim}_{H}\left(\pi_{V}\left(\Phi\left(\Sigma^{\omega}\right)\right) \backslash S\right)\right\} \leq \max \left\{1-\epsilon / 4, \operatorname{dim}_{H}\left(\pi_{V}(\Phi(\Sigma)) \backslash S\right)\right\} .
$$

But this is a deterministic statement, so we conclude that $\operatorname{dim}_{H}\left(\pi_{V}(\Phi(\Sigma)) \backslash S\right)=$ 1.

\section{SeCtions of SELF-Similar Sets AND PERCOLAtion}

Next we obtain a weak dimension conservation property for the lower boxcounting dimension of sections for self-similar sets with dense rotations (Theorem 3.2). We also do so for the Hausdorff dimension of sections of Mandelbrot percolation sets (Theorem 3.4.

The best known model of fractal percolation is Mandelbrot percolation, based on a decomposition of the $d$-dimensional cube into $M^{d}$ equal subcubes of sides $M^{-1}$; its topological properties have been studied extensively, see [3, 6, 24. Nevertheless, statistically self-similar subsets of any self-similar set may be constructed using a similar percolation process which may be set up in terms of the symbolic space formulation of Section 2 ,

Let $\mathcal{I}=\left\{f_{1}, \ldots, f_{m}\right\}$ be an IFS of similarities with attractor $K$. Intuitively, percolation on $K$ is performed by retaining or deleting components of the natural hierarchical construction of $K$ in a self-similar random manner. Starting with some non-empty compact set $D$ such that $f_{i}(D) \subset D$ for all $i$, we select a subfamily of the sets $\left\{f_{1}(D), \ldots, f_{m}(D)\right\}$ according to some probability distribution, and write $K^{1}$ for the union of the selected sets. Then, for each selected $f_{i}(D)$, we choose subsets from $\left\{f_{i} f_{1}(D), \ldots, f_{i} f_{m}(D)\right\}$ according to the same probability distribution, independently for each $i$, with the union of these sets comprising $K^{2}$. Continuing in this way, we get a nested hierarchy $K \supset K^{1} \supset K^{2} \supset \cdots$ of random compact sets, where $K^{k}$ denotes the union of the components remaining at the $k$ th stage. The random percolation set $K^{\omega} \subset K$ is then given by $K^{\omega}=\cap_{k=0}^{\infty} K^{k}$, see Figure 1 .

More formally, percolation on a self-similar set $K$ is defined using the natural representation of $K$ by symbolic space. As in Section 2 we take $\Lambda=\{1, \ldots, m\}$ with $\Sigma_{*}=\cup_{n \geq 0} \Lambda^{n}$ the set of finite words and $\Sigma=\Lambda^{\mathbb{N}}$ the infinite words. The canonical 

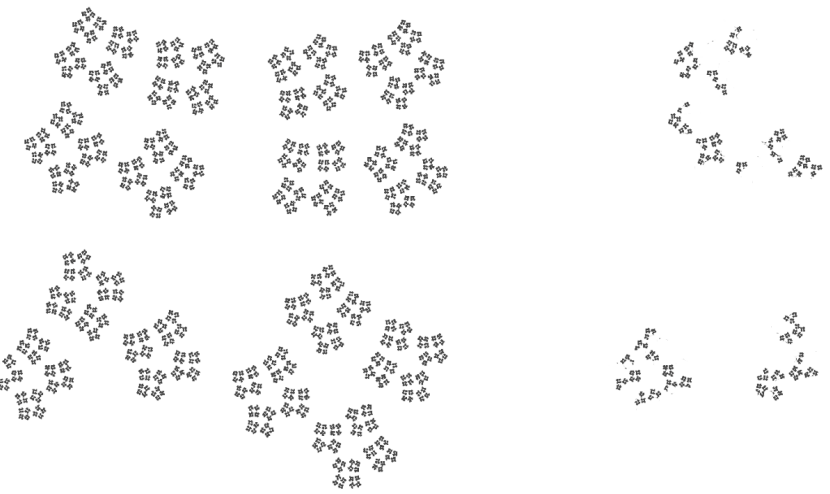

FiguRE 1. A self-similar attractor of an IFS with rotations and a subset obtained by the percolation process

$\operatorname{map} \Phi: \Sigma \rightarrow K \subset \mathbb{R}^{d}$ is given by $\Phi\left(i_{1} i_{2} \ldots\right)=\cap_{n=0}^{\infty} f_{i_{1}} \cdots f_{i_{n}}(D)$ for any nonempty compact set $D$ such that $f_{i}(D) \subset D$ for $i=1, \ldots, m$. Then $K=\cup_{\mathbf{i} \in \Sigma} \Phi(\mathbf{i})$, with $\Phi$ providing a (not necessarily injective) index to the points of $K$.

To define percolation on $K$, let $(\Omega, \mathcal{A}, \mathbb{P})$ be a probability space. Let $X \equiv$ $\left(X_{1}, \ldots, X_{m}\right)$ be a random vector taking values in $\{0,1\}^{m}$. Let $\mathcal{X}=\left\{X^{\mathbf{i}} \equiv\right.$ $\left.\left(X_{1}^{\mathbf{i}}, \ldots, X_{m}^{\mathbf{i}}\right)\right\}_{\mathbf{i} \in \Sigma_{*}}$ be a family of independent random vectors with values in $\{0,1\}^{m}$, each having the distribution of $X$, on the probability space $\left(\Omega^{\Sigma_{*}}, \mathcal{A}_{\mathcal{X}}, \mathbb{P}^{\otimes \Sigma_{*}}\right)$, where $\mathcal{A}_{\mathcal{X}} \subset \mathcal{A}^{\Sigma_{*}}$ is the $\sigma$-algebra generated by $\mathcal{X}$. This defines a random set $\Sigma^{\omega}=\left\{i_{1} i_{2} \ldots \in \Sigma: X_{i_{1}}^{\emptyset} X_{i_{2}}^{i_{1}} X_{i_{3}}^{i_{1} i_{2}} \ldots=1\right\} \subset \Sigma$. The percolation set $K^{\omega} \subset K$ is the image of $\Sigma^{\omega}$ under the canonical map, that is the random set $K^{\omega}=\Phi\left(\Sigma^{\omega}\right)$.

By standard branching process theory [1], if $\mathbb{E}\left(\# i: X_{i}=1\right)>1$ there is a positive probability that $\Sigma^{\omega}$, and thus $K^{\omega}$, is non-empty. Provided the IFS defining $K$ satisfies OSC then, conditional on $K^{\omega} \neq \emptyset$,

$$
\operatorname{dim}_{B} K^{\omega}=\operatorname{dim}_{H} K^{\omega}=s \text { a.s where } s \text { satisfies } \mathbb{E}\left(\sum_{i=1}^{m} X_{i} r_{i}^{s}\right)=1,
$$

where $r_{i}$ is the scaling ratio of $f_{i}$, see [5, 19 .

We say that the percolation process is standard with exponent $\alpha$ if the distribution of $X=\left(X_{1}, \ldots, X_{m}\right)$ is defined by $\mathbb{P}\left(X_{i}=1\right)=r_{i}^{\alpha}, \mathbb{P}\left(X_{i}=0\right)=1-r_{i}^{\alpha}$ independently for $i=1, \ldots, m$. Then by (3.1), provided that $\alpha<\operatorname{dim}_{H} K$, there is a positive probability that $K^{\omega} \neq \emptyset$, in which case $\operatorname{dim}_{H} K^{\omega}=\operatorname{dim}_{H} K-\alpha$ a.s..

The following theorem on the dimension of projections of percolation subsets of self-similar sets was obtained as a corollary of a more general theorem on projections of random cascade measures on self-similar sets [7].

Theorem 3.1. 7] Let $K$ be the attractor of an IFS of contracting similarities on $\mathbb{R}^{d}$ with dense rotations and satisfying OSC. Let $\mathbb{P}$ be a probability distribution of a standard percolation process on $K$ with $\mathbb{E}\left(\# i: X_{i}=1\right)>1$, so that the percolation set $K^{\omega} \neq \emptyset$ with positive probability. Then, conditional on $K^{\omega} \neq \emptyset$, almost surely

$$
\operatorname{dim}_{H} \pi_{V}\left(K^{\omega}\right)=\min \left(k, \operatorname{dim}_{H} K^{\omega}\right),
$$

for every $k$-dimensional subspace $V$. 
Thus, conditional on non-extinction, the projections of $K^{\omega}$ onto all subspaces have the 'generic' dimension. We now apply Proposition 2.2 to sections of selfsimilar sets. The conclusion applies to self-similar sets $K$ such that their projection onto each line is the same as that of the convex hull of $K$. This includes the case where $K$ is connected as well as many other self-similar sets, see Figure 2.
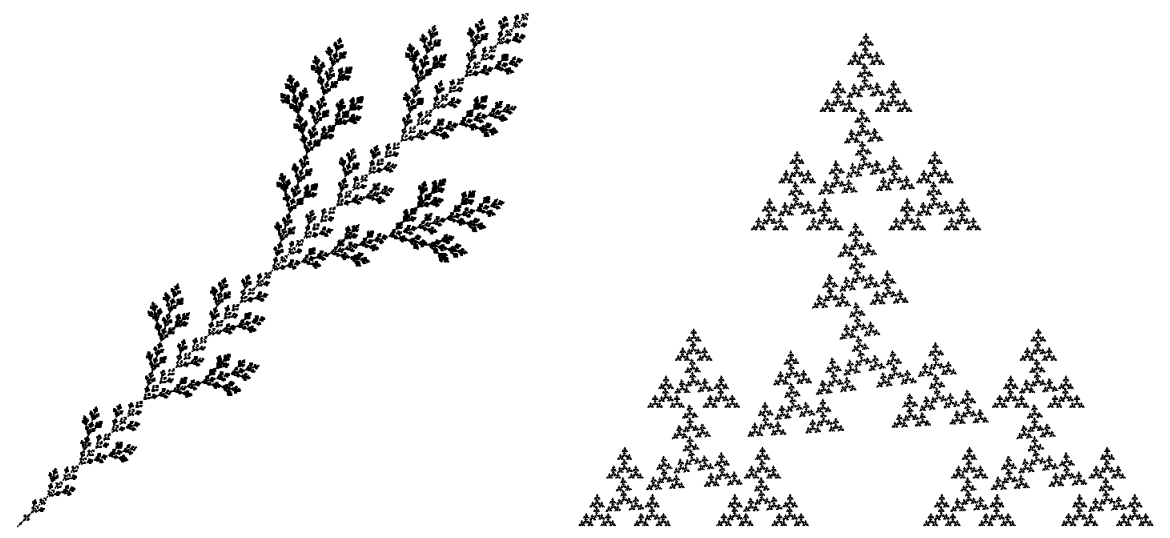

Figure 2. A connected and a totally disconnected self-similar set with dense rotations satisfying the conditions of Theorem 3.2

Theorem 3.2. Let $\mathcal{I}$ be an IFS of contracting similarities on $\mathbb{R}^{d}$ with dense rotations and satisfying OSC. Let $K$ be the attractor of $\mathcal{I}$ and suppose $s=\operatorname{dim}_{H} K>1$ and that the projection of $K$ onto every 1-dimensional subspace equals that of its convex hull. Then for every 1-dimensional subspace $V$ of $\mathbb{R}^{d}$ and all $\epsilon \in(0, s-1)$,

$$
\operatorname{dim}_{H}\left\{x \in V: \underline{\operatorname{dim}}_{B}\left(K \cap \pi_{V}^{-1}(x)\right)>\operatorname{dim}_{H} K-1-\epsilon\right\}=1 .
$$

Proof. Let $K$ have its symbolic representation $\Phi: \Sigma \rightarrow \mathbb{R}^{d}$. As $\Phi[\mathbf{i}]$ is similar to $K$ for all $\mathbf{i} \in \Sigma_{*}$, the projection of each $\Phi[\mathbf{i}]$ onto every 1-dimensional subspace is the same as that of its convex hull. We set up standard percolation with exponent $s-1$ on $K$ via its symbolic representation, as above. Then there is a positive probability of non-extinction, conditional on which almost surely, $\operatorname{dim}_{H} \pi_{V}\left(K^{\omega}\right)=$ $\min \left\{1, \operatorname{dim}_{H} K-(s-1)\right\}=1$ for every line $V$, using Theorem 3.1

A consequence of OSC is that $\Phi$ satisfies conditions (1) and (2) (at 2.1) and (2.2p) with $c_{0}=|K|$ and $c_{1}=\max _{1 \leq i \leq m} r_{i}^{-1}$. Moreover, if $i_{1} \ldots i_{k} \in \Lambda_{\rho}$ then $\mathbb{P}\left(i_{1} \ldots i_{k} \in \Lambda_{\rho}^{\omega}\right)=r_{i_{1}}^{\alpha} \cdots r_{i_{k}}^{\alpha} \leq|K|^{-\alpha} \rho^{\alpha}$, so that $2.5 \mid$ is satisfied. The conclusion follows by Proposition 2.2 since $\Phi(\Sigma)=K$.

It would be desirable to dispense with the requirement in Theorem 3.2 that the projections of $K$ are the same as those of its convex hull. Without such a condition it is not hard to show that $(3.2)$ can be replaced by the conclusion that

$$
\operatorname{dim}_{H}\left\{x \in V: d(x)>\operatorname{dim}_{H} K-1-\epsilon\right\}=1
$$

where $d(x):=\varliminf_{\rho \rightarrow 0} \log \# N_{\rho}\left(L_{x}^{\rho}\right) /-\log \rho$ and where $N_{\rho}\left(L_{x}^{\rho}\right)$ denotes the number of $\mathbf{i} \in \Lambda_{\rho}$ such that $B(\Phi[\mathbf{i}]) \cap L_{y} \neq \emptyset$ for some $y \in[x-\rho, x+\rho]$. (Here $d(x)$ is a kind of lower box-counting dimension conditioning on fibres that is always no less 
than the actual lower box-counting dimension of the fibre, with possibility of being strictly larger.)

Next we apply Proposition 2.1 to Mandelbrot percolation. Let $K$ be the unit cube in $\mathbb{R}^{d}$. Fix an integer $M \geq 2$ and a probability $0<p<1$. We divide $K$ into $M^{d}$ subcubes of side $1 / M$ in the natural way, and retain each subcube independently with probability $p$ to get a set $K^{1}$ formed as a union of the retained subcubes. We repeat this process with the cubes in $K^{1}$, dividing each into $M^{d}$ subcubes of side $1 / M^{2}$ and choosing each with probability $p$ to get a set $K^{2}$, and so on. This process, termed Mandelbrot percolation, leads to a percolation set, which we write here as $K_{p}^{\omega}=\cap_{k=0}^{\infty} K^{k}$ to emphasize the dependence on $p$.

Of course, this may be regarded as percolation on the self-similar set defined by the IFS $\mathcal{I}=\left\{f^{j_{1}, \ldots, j_{d}}: 1 \leq j_{1}, \ldots, j_{d} \leq M\right\}$ on $\mathbb{R}^{d}$ where

$$
f^{j_{1}, \ldots, j_{d}}\left(x_{1}, \ldots, x_{d}\right)=\left(\frac{x_{1}+j_{1}-1}{M}, \ldots, \frac{x_{d}+j_{d}-1}{M}\right) ;
$$

as before the random construction may be represented in symbolic space, using an alphabet of $M^{d}$ letters.

If $p>M^{-d}$ then, as above, that there is a positive probability that $K_{p}^{\omega} \neq \emptyset$, conditional on which $\operatorname{dim}_{H} K_{p}^{\omega}=d+\log p / \log M$. A useful observation is that for $0<p, p^{\prime}<1$ the intersection of independent realizations of the two random sets $K_{p}^{\omega}$ and $K_{p^{\prime}}^{\omega}$ has the same distribution as that of $K_{p p^{\prime}}^{\omega}$.

Rams and Simon 22, 23, 24 and Simon and Vágó 26 have recently obtained results on the dimensions and Lebesgue measure of projections of Mandelbrot percolation that are almost surely valid for projections onto all subspaces.

Theorem 3.3. [22, 26] Let $1 \leq k \leq d-1$ and let $K_{p}^{\omega} \subset \mathbb{R}^{d}$ be the random set obtained by Mandelbrot percolation on the d-dimensional unit cube, using repeated subdivision into $M^{d}$ subcubes, and selecting cubes independently with probability $p>1 / M^{d-k}$. Then, conditional on $K_{p}^{\omega} \neq \emptyset, \operatorname{dim}_{H} K_{p}^{\omega}=d+\log p / \log M>k$, and for every $k$-dimensional subspace $V$ we have $\mathcal{L}^{k}\left(\pi_{V} K_{p}^{\omega}\right)>0$, indeed, $\pi_{V} K_{p}^{\omega}$ contains an open subset of $V$.

Applying Proposition 2.1 to Theorem 3.3 we obtain dimension conservation properties for Mandelbrot percolation.

Theorem 3.4. Let $1 \leq k \leq d-1$. Let $K_{p}^{\omega} \subset \mathbb{R}^{d}$ be the random set obtained by Mandelbrot percolation on the d-dimensional unit cube, using repeated subdivision into $M^{d}$ subcubes and selecting cubes independently with probability $p>1 / M^{d-k}$. For all $\epsilon>0$, almost surely conditional on $K_{p}^{\omega} \neq \emptyset$, for all $k$-dimensional subspaces $V$,

$$
\mathcal{L}^{k}\left\{x \in V: \operatorname{dim}_{H}\left(K_{p}^{\omega} \cap \pi_{V}^{-1}(x)\right) \geq \operatorname{dim}_{H} K_{p}^{\omega}-k-\epsilon\right\}>0 .
$$

Proof. We may represent the heierarchy of $M$-ary subcubes of the unit cube in symbolic space $\Sigma$ with an alphabet $\Lambda$ of $m=M^{d}$ letters with $\Phi: \Lambda \rightarrow K=[0,1]^{d}$ the natural cannonical mapping. With notation for percolation as above, let the probability distribution $\left(X_{1}, \ldots, X_{m}\right)$ on $\Lambda$ be given by $\mathbb{P}\left(X_{i}=1\right)=p, \mathbb{P}\left(X_{i}=0\right)=$ $1-p$, independently for $i=1, \ldots, m$. This defines a random set $\Sigma_{p}^{\omega} \subset \Sigma$ such that $K_{p}^{\omega}=\Phi\left(\Sigma_{p}^{\omega}\right)$ is the Mandelbrot percolation set, with $\operatorname{dim}_{H} K_{p}^{\omega}=d+\log p / \log M$ conditional on non-extinction. Now let $p^{\prime}=p^{-1} M^{-(d-k-\epsilon)}$ and let $\Sigma_{p^{\prime}}^{\omega^{\prime}} \subset \Sigma$ be an independent random set defined in the same way but using probability $p^{\prime}$; we use $\Sigma_{p^{\prime}}^{\omega^{\prime}}$ to 'probe' the dimensions of $K_{p}^{\omega}$. 
The random set $\Sigma_{p}^{\omega} \cap \Sigma_{p^{\prime}}^{\omega^{\prime}}$ has the same distribution as a random set $\Sigma_{p p^{\prime}}^{\omega^{\prime \prime}}$, constructed in the same way with probability $p p^{\prime}$. Thus, conditional on $\Sigma_{p}^{\omega} \cap \Sigma_{p^{\prime}}^{\omega^{\prime}} \neq \emptyset$, $\operatorname{dim}_{H} \Phi\left(\Sigma_{p}^{\omega} \cap \Sigma_{p^{\prime}}^{\omega^{\prime}}\right)=d+\log p p^{\prime} / \log M=k+\epsilon$ almost surely, so by Theorem 3.3 . almost surely,

$$
\mathcal{L}^{k}\left(\pi_{V}\left(\Phi\left(\Sigma_{p}^{\omega} \cap \Sigma_{p^{\prime}}^{\omega^{\prime}}\right)\right)>0\right.
$$

for all $k$-dimensional subspaces $V$. Using independence and Fubini's theorem, conditional on $\Sigma_{p}^{\omega} \neq \emptyset$, almost surely conditional on $\Sigma_{p}^{\omega} \cap \Sigma_{p^{\prime}}^{\omega^{\prime}} \neq \emptyset$, inequality 3.3 holds for all $V$ (Note that, conditional on $\Sigma_{p}^{\omega} \neq \emptyset, \mathbb{P}\left(\Sigma_{p}^{\omega} \cap \Sigma_{p^{\prime}}^{\omega^{\prime}} \neq \emptyset\right)>0$.)

We may regard $\Sigma_{p^{\prime}}^{\omega^{\prime}}$ as an $\alpha$-random subset of $\Sigma$ where $\alpha=-\log p^{\prime} / \log M=$ $\log p / \log M+d-k-\epsilon$. Taking $A=\Sigma_{p}^{\omega}$ in Proposition 2.1 (so in the notation there $\left.A^{\omega}=\Sigma_{p}^{\omega} \cap \Sigma_{p^{\prime}}^{\omega^{\prime}}\right)$ we conclude that, conditional on $\Sigma_{p}^{\omega} \neq \emptyset$,

$$
\mathcal{L}^{k}\left\{x \in V: \operatorname{dim}_{H}\left(\Phi\left(\Sigma_{p}^{\omega}\right) \cap \pi_{V}^{-1}(x)\right) \geq \alpha\right\}>0,
$$

and the conclusion follows, noting that $\Phi\left(\Sigma_{p}^{\omega}\right)=K_{p}^{\omega}$.

\section{Absolute continuity of projections of RAndom SElF-Similar MEASURES}

We now show that we have weak dimension conservation for the Hausdorff dimension of sections of plane self-similar sets in all directions apart from a set of directions of Hausdorff dimension 0 (Theorem 4.8). To achieve this we use Proposition 2.1 together with a result on the absolute continuity of projections of a class of random measures supported by random subsets of self-similar sets (Theorem 4.5), which is a extension of a result of Shmerkin and Solomyak 25] for deterministic measures. We do this first for self-similar sets where the defining similarities are translates of each other. Then a device of Peres and Shmerkin 21] enables us to extend the conclusion to general similarities.

Let

$$
\mathcal{I}=\left\{f_{i}=r R_{\theta} \cdot+a_{i}\right\}_{i=1}^{m}
$$

be an IFS in the plane, where $r \in(0,1)$ and $R_{\theta}$ is the orthogonal rotation with an angle $\theta \in[0,2 \pi)$. As before $\Phi: \Sigma \mapsto \mathbb{R}^{2}$ is the canonical mapping from the symbolic space to the plane.

Let $(\Omega, \mathcal{A}, \mathbb{P})$ be a probability space. Let

$$
X: \Omega \mapsto\left\{\left(p_{1}, \ldots, p_{m}\right) \in[0,1]^{m}: \sum_{i=1}^{m} p_{i}=1\right\}
$$

be a random probability vector allowing zero entries. For $n \in \mathbb{N}$ denote by

$$
\chi_{n}: \Omega^{\mathbb{N}} \rightarrow \Omega
$$

the projection from $\Omega^{\mathbb{N}}$ onto its $n$th coordinate. Then $\mathcal{X}=\left\{X^{(n)}=X \circ \chi_{n}\right\}_{n \in \mathbb{N}}$ forms a i.i.d. sequence on the probability space $\left(\Omega^{\mathbb{N}}, \mathcal{A}_{\mathcal{X}}, \mathbb{P}^{\otimes \mathbb{N}}\right)$, where $\mathcal{A}_{\mathcal{X}} \subset \mathcal{A}^{\otimes \mathbb{N}}$ is the $\sigma$-algebra generated by $\mathcal{X}$. Let $\nu$ be the random probability measure on $\Sigma$ defined by

$$
\nu\left(\left[i_{1} \ldots i_{k}\right]\right)=X_{i_{1}}^{(1)} \cdots X_{i_{k}}^{(k)} \text { for all } i_{1} \ldots i_{k} \in \Sigma_{*} \text {. }
$$


Remark 4.1. Note that the measure $\nu$ is not the same as the random cascade measures studied, for example, in [7]. Here for $k \geq 1$ the ratio $\nu\left(\left[i_{1} \ldots i_{k} i_{k+1}\right]\right)$ : $\nu\left(\left[i_{1} \ldots i_{k}\right]\right)$ is the same for all $i_{1} \ldots i_{k} \in \Lambda^{k}$. The reason why we consider this particular random measure is that its Fourier transform has a convolution structure, which is essential for the proof of absolute continuity in Theorem 4.5.

Let $\mathbb{Q}$ be the probability measure on the product space $\Sigma \times \Omega^{\mathbb{N}}$ given by

$$
\mathbb{Q}(A)=\int_{\Omega^{\mathbb{N}}} \int_{\Sigma} \mathbf{1}_{A}(\mathbf{i}, \boldsymbol{\omega}) \nu(\mathrm{d} \mathbf{i}) \mathbb{P}^{\otimes \mathbb{N}}(\mathrm{d} \boldsymbol{\omega}) \text { for all } A \in \mathcal{B}_{\Sigma} \otimes \mathcal{A}_{\mathcal{X}}
$$

Denote by $\sigma: \Sigma \times \Omega^{\mathbb{N}} \mapsto \Sigma \times \Omega^{\mathbb{N}}$ the left shift

$$
\sigma\left(i_{1} i_{2} \ldots, \omega_{1} \omega_{2} \ldots\right)=\left(i_{2} i_{3} \ldots, \omega_{2} \omega_{3} \ldots\right) .
$$

The next proposition and theorem are direct analogues of those obtained in [7] for random cascade measures.

Proposition 4.2. The dynamical system $\left(\Sigma \times \Omega^{\mathbb{N}}, \mathcal{B}_{\Sigma} \otimes \mathcal{A}_{\mathcal{X}}, \sigma, \mathbb{Q}\right)$ is mixing.

Proof. The proof is similar to that of [7, Proposition 2.2]. Let $\mathcal{B}$ be the semialgebra of $\mathcal{B}_{\Sigma} \otimes \mathcal{A}_{\mathcal{X}}$ consisting of sets of the form

$$
\left\{(\mathbf{i}, \boldsymbol{\omega}):\left.\mathbf{i}\right|_{k}=\mathbf{j}, X_{a}^{(b)} \in B_{a}^{b}\right\}
$$

for $k \in \mathbb{N}, \mathbf{j} \in \Lambda^{k}, b \in\{1, \ldots, k\}, a \in \Lambda$ and $B_{a}^{b}$ Borel subsets of [0,1]. It is clear that $\mathcal{B}$ generates $\mathcal{B}_{\Sigma} \otimes \mathcal{A}_{\mathcal{X}}$, so we only need to verify that $\lim _{n \rightarrow \infty} \mathbb{Q}\left(\sigma^{-n}(A) \cap\right.$ $B)=\mathbb{Q}(A) \mathbb{Q}(B)$ for $A, B \in \mathcal{B}$. This follows since by the construction of $\mathcal{B}$, given $A, B \in \mathcal{B}$, there exists a positive integer $n_{0}$ such that $\sigma^{-n}(A)$ and $B$ are independent for all $n \geq n_{0}$.

Let $\pi_{\beta}: \mathbb{R}^{2} \mapsto \mathbb{R}^{2}$ be orthogonal projection onto the line making an angle $\beta$ with the $x$-axis. Write $\mu=\Phi \nu$ for the measure defined by $\mu(A)=\nu\left(\Phi^{-1} A\right)$. Starting from Proposition 4.2 and proceeding just as in [7, we obtain the following projection property.

Theorem 4.3. Suppose that $\theta / \pi$ is irrational. Then almost surely, for all $\beta \in[0, \pi)$

$$
\operatorname{dim}_{H} \pi_{\beta} \mu=\min \left(1, \operatorname{dim}_{H} \mu\right) .
$$

Proof. When $\theta / \pi$ is irrational, the closed rotation group $G$ generated by $R_{\theta}$ is the whole group $S O(2, \mathbb{R})$. Given this, the proof follows exactly the same lines as in of [7, Sections $2.7 \& 4]$. In particular, since $G=S O(2, \mathbb{R})$, the dimension of the projections equals the maximal possible value, just as in [7, Corollary 4.6],

Theorem 4.5 below, a random analogue of [25. Theorem B], gives conditions for the projections of the random measure $\mu$ to be almost surely absolutely continuous in all directions except for a set $E$ of Hausdorff dimension 0 . First, in the following lemma, we specify the set $E$ and verify that its dimension is 0 . We adapt the delicate estimates of [25, Lemmas $3.2 \& 3.4$ ] to our requirements, in particular obtaining estimates for the dimensions of $E_{q, k}(\delta, N)$ that do not depend on $q$ or $k$.

For $x \in \mathbb{R}$ let $\|x\|=\min \{|x-j|: j \in \mathbb{Z}\}$ and we write $[N]=\{1, \ldots, N\}$ for each positive integer $N$. 
Lemma 4.4. Fix $r \in(0,1), \gamma \in \mathbb{R}, b \in(0, \infty)$ and $\theta \in \mathbb{R}$ with $\theta / \pi$ irrational. For $\delta \in\left(0, \frac{1}{2}\right)$ and integers $q, k \geq 1, N \geq 2$, let $E_{q, k}(\delta, N)$ be the set of all $\beta \in[0, \pi)$ such that

$$
\max _{\tau \in\left[1, r^{-q k}\right]} \frac{1}{N} \#\left\{n \in[N]:\left\|b \tau r^{q-q k(N-n)} \cos (\beta+\gamma-n q k \theta)\right\| \leq \frac{r^{2 q k}}{15}\right\}>1-\delta,
$$

and let

Then $\operatorname{dim}_{H} E=0$.

$$
E=\bigcap_{i \geq 3} \bigcup_{q, k \geq 1} \limsup _{N \rightarrow \infty} E_{q, k}(1 / i, N)
$$

Proof. For the time being we fix the integers $q, k, N \geq 1$ and abbreviate $c:=b r^{q}$, $\ell:=r^{-q k}$ and $\alpha:=q k \theta$. Note that $r^{2 q k} / 15=1 /\left(15 \ell^{2}\right)$. Let $\tau \in[1, \ell]$.

Given $\beta \in[0, \pi)$, for each $n=1, \ldots, N$ write

$$
c \tau \ell^{N-n} \cos (\beta+\gamma-n \alpha)=k_{n}+\epsilon_{n}, \text { where } k_{n} \in \mathbb{Z} \text { and } \epsilon_{n} \in[-1 / 2,1 / 2) .
$$

For $x \in \mathbb{R}$ let $w_{x}=(\cos x, \sin x)$. Since $\alpha / \pi$ is irrational, the unique solution of the equation

$$
c_{1} w_{2 \alpha}+c_{2} w_{\alpha}=w_{0},
$$

is $c_{1}=-1$ and $c_{2}=2 \cos \alpha$. Clearly $\left|c_{1}\right|,\left|c_{2}\right| \leq 2$.

Applying the formula $\left\langle w_{x}, w_{\beta+\gamma-n \alpha}\right\rangle=\cos (\beta+\gamma-n \alpha-x)$ for $x=2 \alpha, \alpha, 0$ and using 4.4 we get that

$$
c_{1} \ell^{2}\left(k_{n+2}+\epsilon_{n+2}\right)+c_{2} \ell\left(k_{n+1}+\epsilon_{n+1}\right)=k_{n}+\epsilon_{n} .
$$

This implies that if

$$
\max \left\{\left|\epsilon_{n}\right|,\left|\epsilon_{n+1}\right|,\left|\epsilon_{n+2}\right|\right\} \leq 1 /\left(15 \ell^{2}\right) \leq 1 /\left(3\left(2 \ell^{2}+2 \ell+1\right)\right),
$$

then

$$
\left|c_{1} \ell^{2} k_{n+2}+c_{2} \ell k_{n+1}-k_{n}\right|<\frac{1}{2},
$$

which means that $k_{n+2}$ and $k_{n+1}$ uniquely determine $k_{n}$. On the other hand,

$$
\left|c_{1} \ell^{2} \epsilon_{n+2}+c_{2} \ell \epsilon_{n+1}-\epsilon_{n}\right| \leq \ell^{2}+\ell+1 .
$$

Hence for fixed $k_{n+2}$ and $k_{n+1}$, there are at most $\left\lfloor 2\left(\ell^{2}+\ell+1\right)+1\right\rfloor \leq 7 \ell^{2}$ possible values of $k_{n}$. Also, from (4.4), there are at most $(2 c \ell+1)\left(2 c \ell^{2}+1\right) \leq(2 b+1)^{2} \ell^{3}$ possible pairs of $\left(k_{N}, k_{N-1}\right)$.

For $\delta \in\left(0, \frac{1}{2}\right)$ denote by $[N]_{\delta}$ the set of all subsets of $[N]$ with cardinality no less than $(1-\delta) N$. For $A \in[N]_{\delta}$ let $\widetilde{A}:=\{0 \leq n \leq N-2: n+2, n+1, n \in A\}$. Then $\# \widetilde{A} \geq(1-3 \delta) N-3$. This implies that the number of possible sequences $\left(k_{n}\right)_{n=0}^{N}$ corresponding to $\beta \in[0, \pi)$ for which $\left|\epsilon_{n}\right| \leq 1 /\left(15 \ell^{2}\right)$ in 4.4 for all $n \in A$, is bounded above by

$$
(2 b+1)^{2} \ell^{3}\left(7 \ell^{2}\right)^{3 \delta N+3} .
$$

Note that once $\left(k_{N}, k_{N-1}\right)$ is given, the possible values of the remaining $k_{n}$ are determined by (4.5), hence the value of $\tau \in[1, \ell]$ is irrelevant. Then, by Chernoff's entropy inequality for binomial sums, see [4], or alternatively using Stirling's approximation,

$$
\#[N]_{\delta} \leq \sum_{p=0}^{\lfloor\delta N\rfloor}\left(\begin{array}{c}
N \\
p
\end{array}\right) \leq 2^{N[-\delta \log \delta-(1-\delta) \log (1-\delta)]} \leq \mathrm{e}^{C \sqrt{\delta} N},
$$

for all $N$ and $\delta \in\left(0, \frac{1}{2}\right)$, where $C$ is a universal constant. 
Combining these estimates, the number of possible sequences $\left(k_{n}\right)_{n=1}^{N}$ corresponding to $\beta \in[0, \pi)$ satisfying

$$
\max _{\tau \in[1, \ell]} \frac{1}{N} \#\left\{n \in[N]:\left\|c \tau \ell^{N-n} \cos (\beta+\gamma-n \alpha)\right\| \leq 1 /\left(15 \ell^{2}\right)\right\}>1-\delta,
$$

is bounded above by

$$
\mathrm{e}^{C \sqrt{\delta} N}(2 b+1)^{2} \ell^{3}\left(7 \ell^{2}\right)^{3 \delta N+3} .
$$

From (4.4), identically

$$
\beta+\gamma-n \alpha=\tan ^{-1}\left(\frac{\ell\left(k_{n+1}+\epsilon_{n+1}\right)}{\left(k_{n}+\epsilon_{n}\right) \sin \alpha}-\cot \alpha\right) .
$$

Since $\alpha / \pi$ is irrational, by estimating the derivatives of the function

$$
f(x)=\tan ^{-1}((\ell / \sin \alpha) x-\cot \alpha),
$$

there is a constant $C^{\prime}$ depending only on $\ell$ and $\alpha$ such that

$$
\beta \in B\left(j \alpha-\gamma+f\left(k_{j+1} / k_{j}\right), C^{\prime} \ell^{-N}\right)
$$

where $j$ may be 1 or 2 (to ensure that $k_{1}$ and $k_{2}$ are not both 0 when $N$ is sufficiently large). Hence the set $E_{q, k}(\delta, N)$ can be covered by

$$
2 \mathrm{e}^{C \sqrt{\delta} N}(2 b+1)^{2} \ell^{3}\left(7 \ell^{2}\right)^{3 \delta N+3}=2 \mathrm{e}^{C \sqrt{\delta} N}(2 b+1)^{2} r^{-3 q k}\left(7 r^{-2 q k}\right)^{3 \delta N+3}
$$

balls of radius $C^{\prime} \ell^{-N}=C^{\prime} r^{q k N}$.

Using these coverings, it follows that

$$
\begin{aligned}
\operatorname{dim}_{H}\left(\limsup _{N \rightarrow \infty} E_{q, k}(\delta, N)\right) & \leq \frac{C \sqrt{\delta}+3 \delta(\log 7-2 q k \log r)}{-q k \log r} \\
& \leq(6+(C+3 \log 7) /-\log r) \sqrt{\delta} .
\end{aligned}
$$

By countable stability of Hausdorff dimension, for $i \geq 3$,

$$
\operatorname{dim}_{H} \bigcup_{q, k \geq 1} \limsup _{N \rightarrow \infty} E_{q, k}(1 / i, N) \leq(6+(C+3 \log 7) /-\log r) / \sqrt{i},
$$

giving the conclusion.

Here is the theorem on the absolute continuity of projections of random measures in all but a small set of exceptional directions when the underlying similarities are translates of each other. The proof uses Fourier transforms along the lines of [25, Theorem B].

Theorem 4.5. Suppose that $\theta / \pi$ is irrational and let $\mathcal{I}$ be an IFS of the form (4.1) satisfying OSC. Then there exists a set $E \subset[0, \pi)$ with $\operatorname{dim}_{H} E=0$ such that, for every random self-similar measure $\mu=\Phi \nu$ with respect to $\mathcal{I}$ of the form defined by (4.2) -4.3 and satisfying

$$
\mathbb{P}\left(\text { there exist } i, j \in \Lambda \text { such that } X_{i}, X_{j} \geq p_{*}\right)=1
$$

for some $p_{*}>0$ and

$$
\mathbb{P}\left(\operatorname{dim}_{H} \mu=s\right)=1
$$

for some $s>1$, almost surely for all $\beta \in[0, \pi) \backslash E$, the projected measure $\pi_{\beta} \mu$ is absolutely continuous with respect to Lebesgue measure. 
Proof. We write $\operatorname{ang}(z)$ for the angle between the line containing $\{0, z\}$ and the $x$-axis. For $i, j \in \Lambda$ let $E_{i, j}$ be the set given by Lemma 4.4 for the ratio $r$ and angle $\theta$ in the IFS (4.1) with $\gamma=\operatorname{ang}\left(a_{i}-a_{j}\right)+\theta$ and $b=\left|a_{i}-a_{j}\right|$. Let $E=\cup_{i, j \in \Lambda} E_{i, j}$. Then $\operatorname{dim}_{H} E=0$; we will show that the projected measures $\pi_{\beta} \mu$ are absolutely continous when $\beta \in[0, \pi) \backslash E$.

With $\mu=\Phi \nu$ as stated, we may, by (4.6), choose $i, j \in \Lambda$ with $\left|a_{i}-a_{j}\right|>0$ such that

$$
\mathbb{P}\left(X_{i}, X_{j} \geq p_{*}\right):=p>0
$$

these $i$ and $j$ will remain fixed throughout the proof.

For each $q \geq 1$, we may regard the attractor $K$ of the IFS (4.1) as the attractor of the iterated IFS

$$
\mathcal{I}_{q}:=\left\{f_{\mathbf{i}}:=f_{i_{1}} \cdots f_{i_{q}} \equiv r^{q} R_{q \theta} \cdot+a_{\mathbf{i}}: \mathbf{i}=i_{1} \ldots i_{q} \in \Lambda^{q}\right\},
$$

so that $K=\Phi_{q}\left(\Sigma_{q}\right)$ where $\Sigma_{q}:=\left\{\mathbf{i}_{1} \mathbf{i}_{2} \ldots: \mathbf{i}_{j} \in \Lambda^{q}\right\}$ and $\Phi_{q}$ is the cannonical map. Let $\nu_{q}$ be the random self-similar measure of the form $(4.2)-(4.3)$ with respect to

$$
\mathcal{X}_{q}=\left\{X^{q,(n)}:=\left(X_{\mathbf{i}}^{q,(n)} \equiv \prod_{l=1}^{q} X_{i_{l}}^{(n q-q+l)}\right)_{\mathbf{i}=i_{1} \ldots i_{q} \in \Lambda^{q}}\right\}_{n \geq 1} .
$$

Then $\mu=\Phi_{q} \nu_{q}$ for all $q \geq 1$. Note that $\mu$ satisfies

$$
\mu=\sum_{\mathbf{i} \in \Lambda^{q}} X_{\mathbf{i}}^{q,(1)} f_{\mathbf{i}} \mu^{q,(1)},
$$

where $\mu^{q,(1)}$ is the copy of $\mu$ generated by $\left\{X^{q,(n+1)}\right\}_{n \in \mathbb{N}}$. In terms of Fourier transforms, writing $T_{q}=r^{q} R_{q \theta}$, equation 4.9 yields that for $\xi \in \mathbb{R}^{2}$,

$$
\widehat{\mu}(\xi)=\sum_{\mathbf{i} \in \Lambda^{q}} X_{\mathbf{i}}^{q,(1)} \mathrm{e}^{\mathrm{i} \pi\left\langle a_{\mathbf{i}}, \xi\right\rangle} \widehat{T_{q} \mu^{q,(1)}}(\xi) .
$$

Iterating 4.10 and taking the limit,

$$
\widehat{\mu}(\xi)=\prod_{n=0}^{\infty} \Psi_{n}^{q}(\xi),
$$

where, for $n \geq 0$,

$$
\Psi_{n}^{q}(\xi)=\sum_{\mathbf{i} \in \Lambda^{q}} X_{\mathbf{i}}^{q,(n+1)} \mathrm{e}^{\mathrm{i} \pi\left\langle T_{q}^{n} a_{\mathbf{i}}, \xi\right\rangle} .
$$

From (4.11), for $q \geq 1$ and $k \geq 2$, we can write $\mu$ as a convolution of two measures $\mu_{q, k} * \eta_{q, k}$, where

$$
\widehat{\mu_{q, k}}(\xi)=\prod_{k \chi n+1} \Psi_{n}^{q}(\xi) \text { and } \widehat{\eta_{q, k}}(\xi)=\prod_{k \mid n+1} \Psi_{n}^{q}(\xi) .
$$

Notice that $\mu_{q, k}$ is within the class of random self-similar measures of the form 4.2-4.3); indeed it has the same law as the random self-similar measure with respect to the IFS

$$
\left\{T_{q}^{k} \cdot+f_{\mathbf{i}_{1}} \cdots f_{\mathbf{i}_{k-1}}((0,0))\right\}_{\mathbf{i}_{1} \ldots \mathbf{i}_{k-1} \in\left(\Lambda^{q}\right)^{k-1}}
$$

and the random vector

$$
\left\{X_{\mathbf{i}_{1}}^{q,(1)} \cdots X_{\mathbf{i}_{k-1}}^{q,(k-1)}\right\}_{\mathbf{i}_{1} \ldots \mathbf{i}_{k-1} \in\left(\Lambda^{q}\right)^{k-1}} .
$$


Thus, almost surely, $\operatorname{dim}_{H} \mu_{q, k}=\frac{k-1}{k} \operatorname{dim}_{H} \mu=\frac{k-1}{k} s>1$ by (4.7) and for some sufficiently large $k$ which we fix for the remainder of the proof. Applying Theorem 4.3 we can find a set $\Omega_{1}$ with $\mathbb{P}\left(\Omega_{1}\right)=1$ such that, for all $\omega \in \Omega_{1}$, for all $\beta \in[0, \pi)$, $q \geq 1$

$$
\operatorname{dim}_{H} \pi_{\beta} \mu_{q, k}=1 .
$$

The rest of the proof estimates the Fourier transform of $\pi_{\beta} \eta_{q, k}$ using Lemma 4.4. From 4.8, for $q \geq 1$ and $n \geq 0$ the event

$$
A_{q, n}=\left\{X_{i}^{(q n+h)}, X_{j}^{(q n+h)} \geq p_{*} \text { for some } h=0, \ldots, q-1\right\}
$$

has probability $\mathbb{P}\left(A_{q, n}\right)=1-(1-p)^{q}$. Since $\left\{\chi_{A_{q, n k}}\right\}_{n \geq 0}$ are i.i.d. random variables for all $q \geq 1$, by the strong law of large numbers we can find a set $\Omega_{2}$ with $\mathbb{P}\left(\Omega_{2}\right)=1$ such that for all $\omega \in \Omega_{2}$, for all $q \geq 1$,

$$
\lim _{N \rightarrow \infty} \frac{1}{N} \sum_{n=0}^{N} \chi_{A_{q, n k}}(\omega)=1-(1-p)^{q} .
$$

By 4.6. we may also find a set $\Omega_{3}$ with $\mathbb{P}\left(\Omega_{3}\right)=1$ such that for all $n \geq 1$,

$$
\text { there exists } \ell \in \Lambda \text { such that } X_{\ell}^{(n)} \geq p_{*} \text {. }
$$

Take $\omega \in \Omega_{1} \cap \Omega_{2} \cap \Omega_{3}$. The rest of the proof will be deterministic.

Let $\beta \in[0, \pi) \backslash E$. By Lemma 4.4 there exists $i_{0}=i_{0}(\beta)$ such that for all $q \geq 1$ there exists $N_{0}=N_{0}(\beta, q)$ such that $\beta \notin E_{q, k}\left(1 / i_{0}, N\right)$ for all $N \geq N_{0}$. In other words, for all $N \geq N_{0}$,

$$
\max _{\tau \in\left[1, r^{-q k}\right]} \frac{1}{N} \#\left\{n \in[N]:\left\|b \tau r^{q-q k(N-n)} \cos (\beta+\gamma-n q k \theta)\right\|>\frac{r^{2 q k}}{15}\right\} \geq \frac{1}{i_{0}},
$$

where $\gamma=\operatorname{ang}\left(a_{i}-a_{j}\right)+\theta$ and $b=\left|a_{i}-a_{j}\right|$. Take $q$ large enough so that $(1-p)^{q}<1 / 4 i_{0}$. We show, in a similar manner to [25, Proposition 3.3], that the projected measure $\pi_{\beta} \eta_{q, k}$ has positive Fourier dimension. (Recall that the Fourier dimension of a measure $\lambda$ is the supremum of $\sigma$ such that $\hat{\lambda}(\xi)=O\left(|\xi|^{-\sigma / 2}\right)$.)

Writing $w_{\beta}=(\cos \beta, \sin \beta)$ as before and applying the formula

$$
\widehat{\pi_{\beta} \lambda}(t)=\widehat{\lambda}\left(t w_{\beta}\right) \quad(t \in \mathbb{R})
$$

for the Fourier transform of the projection of a measure $\lambda$ on $\mathbb{R}^{2}$, we obtain

$$
\widehat{\pi_{\beta} \eta_{q, k}}(t)=\prod_{n=1}^{\infty} \Psi_{k n-1}^{q}\left(t w_{\beta}\right) .
$$

By 4.13), we can find an integer $N_{1}$ such that for all $N \geq N_{1}$,

$$
\sum_{n=0}^{N} \chi_{A_{q, n k}}(\omega) \geq N\left(1-2(1-p)^{q}\right) \geq N\left(1-1 / 2 i_{0}\right) .
$$

We claim that if $\chi_{A_{q, n k}}(\omega)=1$, then there exist distinct $\mathbf{i}_{1}, \mathbf{i}_{2} \in \Lambda^{q}$ such that

$$
X_{\mathbf{i}_{1}}^{q,(k n)}, X_{\mathbf{i}_{2}}^{q,(k n)} \geq\left(p_{*}\right)^{q} ; \quad \operatorname{ang}\left(a_{\mathbf{i}_{1}}-a_{\mathbf{i}_{2}}\right)=\operatorname{ang}\left(a_{i}-a_{j}\right) ; \quad\left|a_{\mathbf{i}_{1}}-a_{\mathbf{i}_{2}}\right|=b r^{q-1} .
$$

To see this, by 4.14 we can find $i_{1}, \ldots, i_{q} \in \Lambda$ such that $X_{i_{l}}^{(q n+l)} \geq p_{*}$ for all $l=0, \ldots, q-1$. Also $X_{i}^{(q k n+h)}, X_{j}^{(q k n+h)} \geq p_{*}$ for some $h \in\{0, \ldots, q-1\}$ since $\chi_{A_{q, k n}}(\omega)=1$. Then it is easy to check that $\mathbf{i}_{1}=i_{1} \ldots i_{h-1} i i_{h+1} \ldots i_{q}$ and $\mathbf{i}_{2}=$ 
$i_{1} \ldots i_{h-1} j i_{h+1} \ldots i_{q}$ satisfy (4.17). Hence for all $n \geq 0$ such that $\chi_{A_{q, n k}}(\omega)=1$ we can write, for some $d_{0}, d_{\mathbf{i}} \in \mathbb{R}$,

$$
\begin{aligned}
\Psi_{n k}^{q}\left(t w_{\beta}\right) & =\sum_{\mathbf{i} \in \Lambda^{q}} X_{\mathbf{i}}^{q,(n k)} \mathrm{e}^{\mathrm{i} \pi\left\langle T_{q}^{n k-1} a_{\mathbf{i}}, t w_{\beta}\right\rangle} \\
& =\mathrm{e}^{\mathrm{i} \pi d_{0}}\left(X_{\mathbf{i}_{1}}^{q,(n k)}+X_{\mathbf{i}_{2}}^{q,(n k)} \mathrm{e}^{\mathrm{i} \pi\left\langle T_{q}^{n k-1}\left(a_{\mathbf{i}_{2}}-a_{\mathbf{i}_{1}}\right), t w_{\beta}\right\rangle}+\sum_{\mathbf{i} \neq \mathbf{i}_{1}, \mathbf{i}_{2}} X_{\mathbf{i}}^{q,(n k)} \mathrm{e}^{\mathrm{i} \pi d_{\mathbf{i}}}\right) .
\end{aligned}
$$

Let $t=\tau\left(r^{-q k}\right)^{N}$, where $\tau \in\left[1, r^{-q k}\right]$ and $N \geq N_{2}:=\max \left\{N_{0}, N_{1}\right\}$, where $N_{0}=N_{0}(\beta, q)$ is given for 4.15). Note that $\operatorname{ang}\left(a_{\mathbf{i}_{1}}-a_{\mathbf{i}_{2}}\right)=\gamma-\theta$. Then

$$
\begin{aligned}
\left\langle T_{q}^{n k-1}\left(a_{\mathbf{i}_{2}}-a_{\mathbf{i}_{1}}\right), t w_{\beta}\right\rangle & =b r^{q-1} t\left\langle T_{q}^{n k-1} w_{-\gamma+\theta}, w_{\beta}\right\rangle \\
& =b \tau r^{q-q k(N-n)} \cos (\beta+\gamma-n q k \theta) .
\end{aligned}
$$

Since $\sum_{\mathbf{i} \in \Lambda^{q}} X_{\mathbf{i}}^{q,(n k)}=1$ and $X_{\mathbf{i}_{1}}^{q,(n k)}, X_{\mathbf{i}_{2}}^{q,(n k)} \geq\left(p_{*}\right)^{q}$, there is a constant $\rho=$ $\rho\left(p_{*}, r, q, k\right)>0$ such that

$$
\left|\Psi_{n k-1}^{q}\left(t w_{\beta}\right)\right| \leq 1-\rho
$$

whenever $\left\|b \tau r^{q-q k(N-n)} \cos (\beta+\gamma-n q k \theta)\right\|>r^{2 q k} / 15$. From 4.15) and 4.16) we deduce that

$$
\#\left\{n \in[N]:\left|\Psi_{n k-1}^{q}\left(t w_{\beta}\right)\right| \leq 1-\rho\right\} \geq\left(\frac{1}{i_{0}}-\frac{1}{2 i_{0}}\right) N=\frac{1}{2 i_{0}} N .
$$

Hence

$$
\left|\widehat{\pi_{\beta} \eta_{q, k}}(t)\right| \leq(1-\rho)^{N / 2 i_{0}} \leq t^{-\log (1-\rho) /\left(2 i_{0} q k \log r\right)},
$$

provided $t \geq r^{-q k\left(N_{2}+1\right)}$, so $\pi_{\beta} \eta_{q, k}$ has positive Fourier dimension.

It was shown in [25, Lemma 4.3] that the convolution of a measure of full Hausdorff dimension with one of positive Fourier dimension is absolutely continuous with respect to Lebesgue measure. Since $\operatorname{dim}_{H} \pi_{\beta} \mu_{q, k}=1$ by (4.12), applying [25, Lemma 4.3] to $\pi_{\beta} \mu_{q, k}$ and $\pi_{\beta} \eta_{q, k}$ gives that $\pi_{\beta} \mu$ is absolutely continuous.

We now apply Theorems 2.1 and 4.5 to get weak dimension conservation for self-similar sets in $\mathbb{R}^{2}$ where the IFS consists of similarities with irrational rotations that are translates of each other and satisfy OSC.

Theorem 4.6. Let $\theta / \pi$ be irrational and suppose that the IFS $\mathcal{I}=\left\{f_{i}=r R_{\theta}\right.$. $\left.+a_{i}\right\}_{i=1}^{m}$ on $\mathbb{R}^{2}$, with $r>1 / m$ and satisfying OSC, has attractor $K$, so that $s:=$ $\operatorname{dim}_{H} K=-\log m / \log r>1$.

Then there is a set $E \subset[0, \pi)$ with $\operatorname{dim}_{H} E=0$ such that for all $\beta \in[0, \pi) \backslash E$, for all $\epsilon \in(0, s-1)$,

$$
\mathcal{L}^{1}\left\{x \in \pi_{\beta}(K): \operatorname{dim}_{H}\left(K \cap \pi_{\beta}^{-1}(x)\right) \geq s-1-\epsilon\right\}>0 .
$$

Proof. For each integer $q>\log 2 /-\log r$ we may regard $K$ as the attractor of the IFS $\mathcal{I}_{q}:=\left\{f_{i_{1}} \cdots f_{i_{q}}: 1 \leq i_{1}, \ldots, i_{q} \leq m\right\}$ so that $K=\Phi_{q}\left(\Sigma_{q}\right)$ where $\Sigma_{q}:=$ $\left\{\mathbf{i}_{1} \mathbf{i}_{2} \ldots: \mathbf{i}_{j} \in \Lambda^{q}\right\}$ and $\Phi_{q}$ is the cannonical map. Let $E_{q} \subset[0, \pi)$ be the set with $\operatorname{dim}_{H} E_{q}=0$ given by that Theorem 4.5 for the IFS $\mathcal{I}_{q}$. Take $E=\cup_{q>\log 2 /-\log r} E_{q}$ so that $\operatorname{dim}_{H} E=0$.

Now fix $\epsilon \in(0, s-1)$. Let $q>\log 2 /-\log r$ be an integer to be specified later. Let

$$
p_{q}:=\left(r^{q(s-1-\epsilon)}-\frac{2}{m^{q}}\right) \frac{m^{q}}{m^{q}-2}=\frac{m^{q(1+\epsilon) / s}-2}{m^{q}-2} \in(0,1),
$$


since $r^{s}=m^{-1}$ and $2<r^{-q}=m^{q / s}$. Let $S_{q}$ be a random subset of $\Lambda^{q}$ defined as follows. First choose two different symbols from $\Lambda^{q}$ with uniform probability, then select each of the remaining $m^{q}-2$ symbols with probability $p_{q}$, all actions being independent; in this way $S_{q}$ always contains at least two symbols. Moreover, for each $\mathbf{i} \in \Lambda^{q}$,

$$
\begin{aligned}
\mathbb{P}\left(\mathbf{i} \in S_{q}\right) & =\frac{2}{m^{q}}+\frac{m^{q}-2}{m^{q}} p_{q} \\
& =r^{q(s-1-\epsilon)} .
\end{aligned}
$$

Let $\left\{S_{q}^{(k)}: k \in \mathbb{N}\right\}$ be a sequence of independent copies of $S_{q}$. Then the set

$$
\Sigma_{q}^{\omega}:=S_{q}^{(1)} \times S_{q}^{(2)} \times \cdots
$$

is an $\alpha$-random set, with $\alpha=\log r^{q(s-1-\epsilon)} / \log r^{q}=s-1-\epsilon$, witn $\Phi_{q}$ satisfying (1) and (2) at (2.1) and (2.2).

Define a random vector $X_{q}$ in a uniform manner, that is,

$$
X_{q}=\left\{\frac{\chi_{\left(\mathbf{i} \in S_{q}\right)}}{\# S_{q}}\right\}_{\mathbf{i} \in S_{q}} ;
$$

then $\left(X_{q}\right)_{\mathbf{i}} \geq 1 / m^{q}:=p_{*}$ for at least two $\mathbf{i} \in S_{q}$. Let $\left\{X_{q}^{(k)}: k \in \mathbb{N}\right\}$ be independent copies of $X_{q}$ which are supported by $S_{q}^{(k)}$. These random vectors define a random measure $\nu_{q}$ on $\Sigma_{q}$ of the form described in 4.2 and 4.3 at the start of this section. Then $\nu_{q}$ has support $\Sigma_{q}^{\omega}$, and $\Phi_{q} \nu_{q}$ has support $K^{\omega}=\Phi_{q}\left(\Sigma_{q}^{\omega}\right)$. From the strong law of large numbers, and using OSC when mapping the measure under $\Phi_{q}$, almost surely

$$
\operatorname{dim}_{H} \Phi_{q} \nu_{q}=\frac{\mathbb{E}\left(\log \# S_{q}\right)}{-\log r^{q}} .
$$

Write $\operatorname{Bin}(n, p)$ to denote the binomial distribution with $n$ points and probability p. Then

$$
\mathbb{E}\left(\log \# S_{q}\right)=\mathbb{E}\left(\log \left[\operatorname{Bin}\left(m^{q}-2, p_{q}\right)+2\right]\right)=\log \left(m^{q(1+\epsilon) / s}\right)-o(1)
$$

as $q \rightarrow \infty$, on using 4.18 to express $p_{q}$ in terms of $m$ together with a simple application of Chebyshev's inequality. Thus

$$
\operatorname{dim}_{H} \Phi_{q} \nu_{q}=\frac{\log \left(m^{q(1+\epsilon) / s}\right)-o(1)}{-\log m^{-q / s}}=1+\epsilon-o\left(q^{-1}\right)>1
$$

provided we now choose $q$ sufficiently large.

From Theorem 4.5. almost surely for all $\beta \in[0, \pi) \backslash E \subset[0, \pi) \backslash E_{q}$, the projected measure $\pi_{\beta} \Phi_{q} \nu_{q}$ is absolutely continuous with respect to Lebesgue measure, so $\mathcal{L}^{1}\left(\pi_{\beta}\left(K^{\omega}\right)\right)>0$. The conclusion follows from Proposition 2.1. taking $A=\Sigma$, $K=\Phi(\Sigma)$ and $\alpha=s-1-\epsilon$.

We now extend Theorem 4.6 to general sets of similarities using a technique of Peres and Shmerkin [21, Proposition 6]. This allows us to reduce a general plane IFS to one where the similarities are mutual translates with the attractor a subset of that of the original IFS and of arbitraily close dimension to which we may apply Theorem 4.6 . 
Proposition 4.7. Let $\mathcal{I}=\left\{f_{i}=r_{i} R_{\theta_{i}} \cdot+a_{i}\right\}_{i=1}^{m}$ be an IFS on $\mathbb{R}^{2}$ satisfying OSC with attractor $K$. For all $\epsilon>0$ there is an IFS $\mathcal{I}_{\epsilon}$, satisfying SSC and formed by a collection of compositions of maps from $\mathcal{I}$, such that all the maps in $\mathcal{I}_{\epsilon}$ have the same rotation $R_{\theta}$ for some angle $\theta$ and the same contraction ratio $0<r<1$, and with attractor $K_{\epsilon} \subset K$ such that $\operatorname{dim}_{H} K_{\epsilon}>\operatorname{dim}_{H} K-\epsilon$.

Moreover, if $\mathcal{I}$ has dense rotations then we may take $\theta / \pi$ to be irrational.

Proof. First we may assume that $\mathcal{I}$ satisfies SSC, since there is an IFS formed by compositions of the maps in $\mathcal{I}$ that satisfies SSC with attractor a subset of $K$ and with Hausdorff dimension arbitrarily close to that of $K$, see, for example, 20].

Next, as in the proof of [21, Proposition 6], we may find integers $n_{1}, \ldots, n_{m}$ such that the IFS $\mathcal{I}_{\epsilon}$ formed by all those compositions of the maps of $\mathcal{I}$ taken in any order such that $f_{i}$ occurs $n_{i}$ times for each $i=1, \ldots, m$, has an attractor $K_{\epsilon} \subset K$ with $\operatorname{dim}_{H} K_{\epsilon}>\operatorname{dim}_{H} K-\epsilon$. All the maps in $\mathcal{I}_{\epsilon}$ have rotation $R_{\theta}=R_{n_{1} \theta_{1}+\cdots+n_{m} \theta_{m}}$ and contraction ratio $r=r_{1}^{n_{1}} \cdots r_{m}^{n_{m}}$.

Now suppose that $\mathcal{I}$ has dense rotations. If $\left(n_{1} \theta_{1}+\cdots+n_{m} \theta_{m}\right) / \pi$ is irrational then there is nothing further to prove. Otherwise, at least one of the $\theta_{i}$, say $\theta_{1}$, is an irrational multiple of $\pi$. By a slight modification of the proof of [21, Proposition $6]$ we may conclude that the attractor of the IFS $\mathcal{I}_{\epsilon}^{\prime}$ formed by the compositions of the maps of $\mathcal{I}$ such that $f_{1}$ occurs $n_{1}-1$ times and $f_{i}$ occurs $n_{i}$ times for $i=$ $2, \ldots, m$, with attractor $K_{\epsilon}^{\prime} \subset K$ has $\operatorname{dim}_{H} K_{\epsilon}^{\prime}>\operatorname{dim}_{H} K-\epsilon$. (We just note in 21, Proposition 6] that the number of paths ending at a neighboring lattice point to $v$ is comparable to the number of paths ending at $v$.) Then $\left(\left(n_{1}-1\right) \theta_{1}+\cdots+n_{m} \theta_{m}\right) / \pi$ is irrational so the conclusion holds for $\mathcal{I}_{\epsilon}^{\prime}$.

Theorem 4.8. Let

$$
\mathcal{I}=\left\{f_{i}=r_{i} R_{\theta_{i}} \cdot+a_{i}\right\}_{i=1}^{m}
$$

be an IFS on $\mathbb{R}^{2}$ with dense rotations satisfying OSC, with attractor $K$ and with $s=\operatorname{dim}_{H} K>1$, where $s$ is given by $\sum_{i=1}^{m} r_{i}^{s}=1$. Then there is a set $E \subset[0, \pi)$ with $\operatorname{dim}_{H} E=0$ such that for all $\beta \in[0, \pi) \backslash E$, for all $\epsilon \in(0, s-1)$,

$$
\mathcal{L}^{1}\left\{x \in \pi_{\beta}(K): \operatorname{dim}_{H}\left(K \cap \pi_{\beta}^{-1}(x)\right) \geq s-1-\epsilon\right\}>0 .
$$

Proof. For each $\epsilon>0$, applying Theorem 4.6 to the amended IFS $\mathcal{I}_{\epsilon}$ with attractor $K_{\epsilon}$ given by Proposition 4.7 (replacing $\epsilon$ by $\epsilon / 2$ in both theorem and proposition), there is a set $E_{\epsilon} \subset[0, \pi)$ with $\operatorname{dim}_{H} E_{\epsilon}=0$, such that 4.19$]$ holds for all $\beta \in E_{\epsilon}$. So that the set of exceptional $\beta$ does not depend on $\epsilon$, we let $E=\cup_{n=n_{0}}^{\infty} E_{2^{-n}}$, where $2^{-n_{0}}<s-1$, so that $\operatorname{dim}_{H} E=0$.

\section{FURTHER REMARKS}

1. A natural question is whether these results can be strengthened from 'weak dimension conservation' to 'dimension conservation', that is whether the ' $\epsilon$ ' can be removed in the conclusion of Proposition 2.2, and in Theorems 3.2, 3.4, 4.6 and 4.8 .

2. Another natural question is whether, in Proposition 2.2, the condition on the projection of $B(\Phi[\mathbf{i}])$ can be weakened, with a consequential weakening of the corresponding condition on the projections of $K$ in Theorem 3.2. Furthermore, can $\underline{\operatorname{dim}}_{B}$ of the sections be replaced by $\operatorname{dim}_{H}$ in the conclusions of Proposition 2.2 and Theorem 3.2? An alternative approach would be to eliminate the exceptional set of directions in Theorem 4.6 and thus Theorem 4.8 . 
This raises the question of whether the box-dimension and Hausdorff dimension of sections of self-similar set are 'typically' equal for all, or perhaps 'nearly all' directions. If $\underline{\operatorname{dim}}_{B}(K \cap L)=\operatorname{dim}_{H}(K \cap L)$ for every line $L$, or at least for a large set of lines, then one might be able to replace lower box dimension by Hausdorff dimension in the conclusion of Theorem 3.2. There are plane self-similar sets defined by homotheties with at least some sections having distinct Hausdorff and lower box dimensions, for example for certain horizontal sections of the 1-dimensional Sierpiński triangle, that is the attractor of the plane IFS with maps $f_{1}(x, y)=$ $\left(\frac{1}{3} x, \frac{1}{3} y\right), f_{2}(x, y)=\left(\frac{1}{3} x+\frac{2}{3}, \frac{1}{3} y\right), f_{3}(x, y)=\left(\frac{1}{3} x, \frac{1}{3} y+\frac{2}{3}\right)$ (we are grateful to Thomas Jordan for pointing out this example to us); see also [2. Is this possible for selfsimilar sets with dense rotations?

3. Similar conclusions to Proposition 2.2 and thus Theorem 3.2 might be expected for projections onto $k$-dimensional subspaces $V$ where $k \geq 2$. However, it seems hard to get an analogue of Lemma 2.3 in this case. One would need to show that for any cube $I \subset V$ with $|I| \leq r$ there is a bounded number of points $x_{i} \in V$ with $N\left(x_{i}, r\right) \leq M$ such that if $N(x, r) \leq M$ for some $x \in I$ then some $L_{x_{i}}$ intersects every set $B(\Phi[\mathbf{i}])$ such that $\mathbf{i} \in \Lambda_{r}$ that intersects $L_{x}$. (Here $N(x, r)$ is the number of $B(\Phi[\mathbf{i}])$ with $\mathbf{i} \in \Lambda_{r}$ that intersect $L_{x}$, the $(d-k)$-plane through $x \in V$ and perpendicular to $V$.)

4. Our results have been presented for self-similar sets defined by orientationpreserving similarities. It would be possible to extend them to allow some of the maps to be orientation-reversing, for example by replacing an IFS by one formed by appropriate orientation-preserving compositions of the maps with little reduction in the dimension of the attractor, as in the proof of [21, Proposition 6].

\section{REFERENCES}

[1] K. B. Athreya and P. E. Ney. Branching processes. Dover Publications Inc., Mineola, NY, 2004.

[2] B. Bárány, A. Ferguson and K. Simon. Slicing the Sierpiński gasket. Nonlinearity, 25: 17531770, 2012.

[3] M. Dekking. Random Cantor sets and their projections. In Fractal Geometry and Stochastics IV, Progr. Probab., 61, pp. 269-284. Birkhäuser Verlag, Basel, 2009.

[4] P. Erdős and J. Spencer. Probabilistic Methods in Combinatorics. Academic Press, New York - London, 1974.

[5] K. J. Falconer. Random fractals. Math. Proc. Cambridge Philos. Soc., 100: 559-582, 1986.

[6] K. J. Falconer. Fractal Geometry - Mathematical Foundations and Applications. John Wiley \& Sons Ltd., Chichester, 3rd Ed., 2014.

[7] K. J. Falconer and X. Jin. Exact dimensionality and projections of random self-similar measures and sets. J. Lond. Math. Soc. (2), electronic version 2014.

[8] H. Furstenberg. Ergodic fractal measures and dimension conservation. Ergodic Theory Dynam. Systems, 28: 405-422, 2008.

[9] M. Hochman. Dynamics on fractals and fractal distributions, arXiv:1008.3731v2, 2013.

[10] M. Hochman and P. Shmerkin. Local entropy averages and projections of fractal measures. Ann. of Math.(2), 175: 1001-1059, 2012.

[11] J. Hutchinson. Fractals and self-similarity. Indiana Univ. Math. J., 30: 713-747, 1981.

[12] R. Kaufman. On Hausdorff dimension of projections. Mathematika, 15: 153-155, 1968.

[13] Q.-H. Liu, L.-F. Xi. and Y.-F. Zhao. Dimensions of intersections of the Sierpinski carpet with lines of rational slopes. Proc. Edinb. Math. Soc. (2), 50: 411-27, 2007.

[14] A. Manning and K. Simon. Dimension of slices through the Sierpinski carpet. Trans. Amer. Math. Soc., 365: 213-250, 2013.

[15] J. M. Marstrand. Some fundamental geometrical properties of plane sets of fractional dimensions. Proc. London Math. Soc.(3), 4: 257-302, 1954. 
[16] J. M. Marstrand. The dimension of Cartesian product sets. Proc. Cambridge Philos. Soc., 50:198-202, 1954.

[17] P. Mattila. Hausdorff dimension, orthogonal projections and intersections with planes. Ann. Acad. Sci. Fenn. Ser. A I Math., 1: 227-244, 1975.

[18] P. Mattila. Geometry of sets and measures in Euclidean spaces, Fractals and rectifiability. Cambridge Studies in Advanced Mathematics 44. Cambridge University Press, Cambridge, 1995.

[19] R. D. Mauldin and S. C. Williams. Random recursive constructions: asymptotic geometric and topological properties. Trans. Amer. Math. Soc., 295: 325-346, 1986.

[20] T. Orponen. On the distance sets of self-similar sets. Nonlinearity, 6: 1919-1929, 2012.

[21] Y. Peres and P. Shmerkin. Resonance between Cantor sets. Ergodic Theory Dynam. Systems., 29: 201-221, 2009 .

[22] M. Rams and K. Simon. The dimension of projections of fractal percolations. J. Stat. Phys., 154: 633-655, 2014.

[23] M. Rams and K. Simon. Projections of fractal percolations. Ergodic Theory Dynam. Systems., to appear, arXiv:1306.3844, 2013.

[24] M. Rams and K. Simon. The geometry of fractal percolation. Proceedings of Advances in Fractals and Related Topics 2012, arXiv:1306.3847, 2013.

[25] P. Shmerkin and B. Solomyak. Absolute continuity of self-similar measures, their projections and concolutions. arXiv:1406.0204 2014.

[26] K. Simon and L. Vágó Projections of Mandelbrot percolation in higher dimensions. Ergodic Theory Dynam. Systems., arXiv:1407.2225, 2014.

Mathematical Institute, University of St Andrews, North Haugh, St Andrews, Fife, KY16 9SS, United Kingdom

E-mail address: kjf@st-andrews.ac.uk

School of Mathematics, Alan Turing Building, The University of Manchester, ManchESTER, M13 9PL, United Kingdom

E-mail address: xiong.jin@manchester.ac.uk 